\title{
Polynomial functors and trees
}

\author{
To my Father for his 70th birthday
}

JOACHIM KOCK

\begin{abstract}
We explore the relationship between polynomial functors and (rooted) trees. In the first part we use polynomial functors to derive a new convenient formalism for trees, and obtain a natural and conceptual construction of the category $\Omega$ of Moerdijk and Weiss; its main properties are described in terms of some factorisation systems. Although the constructions are motivated and explained in terms of polynomial functors, they all amount to elementary manipulations with finite sets. In the second part we describe polynomial endofunctors and monads as structures built from trees, characterising the images of several nerve functors from polynomial endofunctors and monads into presheaves on categories of trees. Polynomial endofunctors and monads over a base are characterised by a sheaf condition on categories of decorated trees. In the absolute case, one further condition is needed, a certain projectivity condition, which serves also to characterise polynomial endofunctors and monads among (coloured) collections and operads.
\end{abstract}

\section{Contents}

$0 \quad$ Introduction and preliminaries 2

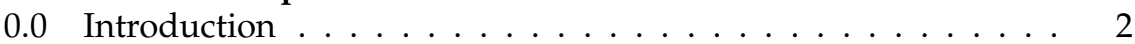

0.1 Polynomial functors $\ldots \ldots \ldots \ldots$

1 Trees in terms of polynomial endofunctors 11

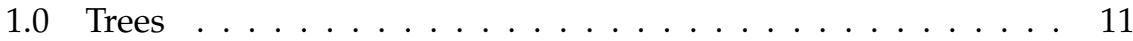

1.1 The category $\mathbf{T E m b} \ldots \ldots \ldots \ldots \ldots \ldots$

1.2 P-trees and free monads . . . . . . . . . . . . . . 20

1.3 The category Tree . . . . . . . . . . . . . . . . . 24

2 Polynomial endofunctors in terms of trees 30

2.0 Background on nerve theorems . . . . . . . . . . 30

2.1 Elements of a polynomial endofunctor . . . . . . . . . . 33

2.2 Generic factorisation, and trees as arities . . . . . . . . . 36

2.3 Sheaf conditions and nerve theorem for slices . . . . . . . . . . 42

2.4 Polynomial endofunctors and collections . . . . . . . . . . 44

2.5 Polynomial monads and operads . . . . . . . . . . . . . . 48 


\section{Introduction and preliminaries}

\subsection{Introduction}

While linear orders and the category $\Delta$ of nonempty finite ordinals constitute the combinatorial foundation for category theory, the theories of operads and multicategories are based on trees. Where $\Delta$ is very well understood and admits good formal descriptions, trees are often treated in an ad hoc manner, and arguments about them are often expressed in more or less heuristic terms based on drawings.

Having solid combinatorial foundations is crucial for developing homotopical and higher-dimensional versions of the theories. Recently, Moerdijk and Weiss [17], [18] have undertaken the project of developing a homotopy theory for operads by mimicking the simplicial approach to homotopical category theory. Their work is the main motivation and inspiration for the present article (although no homotopy theory is developed here).

This paper analyses the relationship between polynomial functors, polynomial monads and trees, keeping the analogy with graphs, categories and linear orders as close as possible. In both cases, the interplay between algebra, combinatorics, and homotopy theory follows the same pattern, whose general theory has been worked out by Weber [21]: categories are first defined algebraically, as algebras for a nice monad on the category of graphs; there is a canonical way to distill the combinatorics of such a monad, which in this case yields the category $\Delta$; finally categories are characterised among presheaves on $\Delta$. Analogously, polynomial monads are algebras for a nice monad on the category of polynomial endofunctors; from this monad a category of trees arises naturally; finally polynomial monads are characterised among presheaves on this category of trees.

The theory of polynomial functors is relatively new and has hitherto mostly been explored from the viewpoint of type theory and computer science (some references can be found in [5] or [12]). The point that polynomial functors are an excellent tool for making explicit and analysing the combinatorics underlying operad theory was first made in the paper [13], where polynomial functors were used to extract the first purely combinatorial characterisation of opetopes. Opetopes can be seen as higherdimensional analogues of trees. The present paper goes to a more fundamental level, substantiating that already the usual notion of tree is of polynomial nature and benefits from this explicitation. The resulting formalism of trees is in fact elementary, has a clear intuitive content, and is easy to work with.

One single observation accounts for the close relationship between polynomial endofunctors and trees, namely that they are represented by diagrams of the same shape, as we now proceed to explain. Although this observation is both natural and fruitful, it seems not to have been made before.

Trees are usually defined and manipulated in either of two ways:

- 'Topological/static characterisation': trees are graphs $E \Longrightarrow V$ with certain topological properties and structure (a base point). 
- 'Recursive characterisation': a tree is either a trivial tree or a collection of smaller trees.

In this work, a different viewpoint is taken, specifically designed for the use of trees in operad theory and related topics:

- 'Operational characterisation': trees are certain many-in/one-out structures, i.e. built from building blocks like

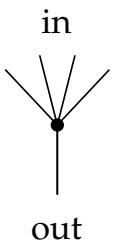

Accordingly a tree should have a set of edges $A$, and a set of vertices (or nodes) $N$, which we think of as operations; these should have inputs and output (source and target). So the structure is something like

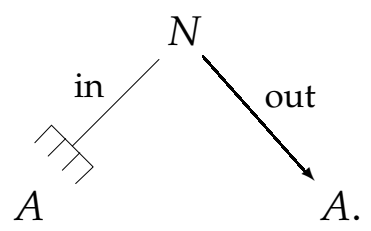

The fork represents a 'multi-valued map', because a node may have several input edges. A standard way to encode multi-valued maps is in terms of correspondences or spans; hence we arrive at this shape of diagram to represent a tree:

$$
A \stackrel{s}{\longleftarrow} M \stackrel{p}{\longrightarrow} N \stackrel{t}{\longrightarrow} A ;
$$

to be explicit, $M$ is the set of all input edges (i.e. pairs $(b, e)$ where $b$ is a node and $e$ is an input edge of $b$ ). In order to be trees, such diagrams should satisfy certain axioms, which turn out to be quite simple.

Although this is clearly also a static graph-like definition, its operational aspect comes to the fore with the observation that this shape of diagram is precisely what defines polynomial endofunctors [12], vindicating the interpretation of $N$ as a set of operations. The polynomial endofunctor represented by a diagram (1) is

$$
\text { Set } / A \stackrel{{ }^{*}}{\longrightarrow} \text { Set } / M \stackrel{p_{*}}{\longrightarrow} \text { Set } / N \stackrel{t_{!}}{\longrightarrow} \text { Set } / A \text {. }
$$

Among all polynomial endofunctors we characterise those that correspond to trees, and since the involved conditions have a clear intuitive content and are convenient to work with, we will simply take this as the definition of tree (1.0.3).

If in (1) the map $p$ is the identity, the diagram is just that of a directed (non-reflexive) graph, and the associated polynomial functor becomes linear. Imposing the tree axioms 
in this case yields linear trees, i.e. finite linear orders. Linear polynomial monads are the same thing as small categories.

Polynomial endofunctors and monads are intimately related with collections and operads (the precise relationship is given in Sections 2.4 and 2.5), but are distinguished by a representability feature: they can be represented by diagrams of sets like (1), and most constructions with polynomial functors can be performed in terms of elementary operations on those representing sets. As a result, all operations on trees can be carried out completely formally (e.g., grafting is given in terms of pushouts of finite sets (1.1.19)), without ever having seen a tree in nature - although of course the arguments are easier to follow with drawings of trees in mind.

The recursive aspect of trees is also prominent in the present approach, remembering that polynomial endofunctors provide categorical semantics for inductive data types ( $W$-types), the latter appearing as initial Lambek algebras for the former [16]. In fact, a recursive characterisation of trees (1.1.21) follows quite easily from the definition. While in type theory trees (of a certain branching profile P) appear as initial algebras for some polynomial functor $\mathrm{P}$ (expressing the branching profile), in this work trees are themselves certain polynomial functors. In a precise sense they are absolute trees, i.e. not relative to any preassigned branching profile.

The paper naturally divides into two parts: the first part concerns the categories of trees. Most arguments in this part are quite elementary, and some of the initial manœuvres may appear pedantic. They are deliberately included in order to emphasise the workability of the new tree formalism - the reader is challenged to provide easier arguments in other formalisms of trees. The second part uses the tree formalism to prove theorems about polynomial functors and polynomial monads and to clarify the relationship with operads. This part is of a more technical nature and requires some more category theory.

We proceed to give an overview of each of the two parts of the paper.

0.0.1. Overview of Part 1: trees in terms of polynomial endofunctors. After recalling the relevant notions from the theory of polynomial functors, we define a tree to be a diagram of sets of shape (1) satisfying four simple conditions (1.0.3). The category TEmb is the full subcategory of PolyEnd (the category of polynomial endofunctors) consisting of the trees. The morphisms are diagrams

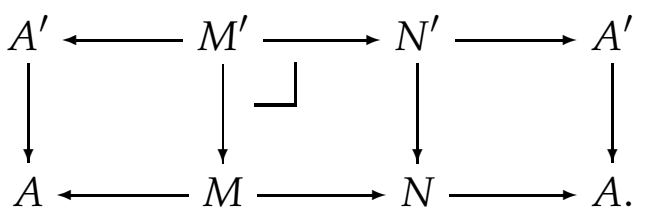

The symbol TEmb stands for 'tree embeddings', as it turns out maps between trees are always injective (1.1.3) and correspond to a notion of subtree. Root-preserving embeddings and ideal embeddings are characterised in terms of pullback conditions, and every tree embedding factors as root-preserving followed by ideal embedding (1.1.15). These two classes of maps allow pushouts along each other in the category TEmb - 
this is grafting of trees (1.1.19). This leads to a recursive characterisation of trees (1.1.21), as well as the useful result that every tree is the iterated pushout of its one-node subtrees over its inner edges (1.1.24).

For a polynomial endofunctor $\mathrm{P}$, a $\mathrm{P}$-tree is a tree with a map to $\mathrm{P}$. This amounts to structuring the set of input edges of each node. For example, if $\mathrm{M}$ is the free-monoid monad (0.1.8), then $\mathrm{M}$-trees are precisely planar trees. It is shown, using the recursive characterisation of trees, that the set of isomorphism classes of P-trees, denoted $\operatorname{tr}(\mathrm{P})$, is the least fixpoint (initial Lambek algebra) for the polynomial endofunctor $1+P$ (Theorem 1.2.5). This leads to the following explicit construction of the free monad on $\mathrm{P}:$ if $\mathrm{P}$ is given by the diagram $A \leftarrow M \rightarrow N \rightarrow A$, then the free monad on $\mathrm{P}$ is given by

$$
A \leftarrow \operatorname{tr}^{\prime}(\mathrm{P}) \rightarrow \operatorname{tr}(\mathrm{P}) \rightarrow A
$$

where $\operatorname{tr}^{\prime}(\mathrm{P})$ denotes the set of isomorphism classes of P-trees with a marked leaf (1.2.8). The monad structure is given by grafting P-trees. We are particularly interested in free monads on trees. Since maps between trees are embeddings, the free monad on a tree $\mathrm{T}=(A \leftarrow M \rightarrow N \rightarrow A)$ is given by

$$
A \leftarrow \operatorname{sub}^{\prime}(\mathrm{T}) \rightarrow \operatorname{sub}(\mathrm{T}) \rightarrow A
$$

(where $\operatorname{sub}(T)$ (resp. sub $(T)$ ) denotes the set of subtrees of $T$ (resp. subtrees with a marked leaf)).

We now define Tree to be the category whose objects are trees and whose arrows are maps between their free monads (1.3.1). In other words, Tree is a full subcategory of PolyMnd (the category of polynomial monads): it is the Kleisli category of $\mathbf{T E m} \boldsymbol{b}$ with respect to the free-monad monad on PolyEnd. (It is shown that any map in PolyEnd between free monads on trees is a monad map (1.3.2).) In explicit terms, morphisms send edges to edges and subtrees to subtrees. The category Tree is equivalent to the category $\Omega$ of Moerdijk and Weiss [17], whose presheaves are called dendroidal sets. Its construction in terms of polynomial functors reveals important properties analogous to properties of $\Delta$. In fact, $\Delta$ is equivalent to the full subcategory in Tree consisting of the linear trees.

The main intrinsic features of the category Tree are expressed in terms of three factorisation systems: Tree is shown to have has surjective/injective factorisation, generic/free factorisation, as well as root-preserving/ideal-embedding factorisation. The generic maps are precisely the boundary-preserving maps, and the generic/free factorisation system plays an important role in the second part of the paper. The compatibilities between the three factorisation systems are summarised in this figure:

\begin{tabular}{|c|c|}
\hline surjective & \multicolumn{2}{c|}{ injective } \\
\hline generic & free \\
\hline root preserving & ideal \\
\hline
\end{tabular}

As suggested by the figure, every arrow factors essentially uniquely as a surjection followed by a generic injection, followed by a free root-preserving map followed by 
an ideal embedding. Explicit descriptions are derived for each of these four classes of maps. The surjections consist in deleting unary nodes, the generic injections are node refinements (and of course the free maps are the tree embeddings). The ideal embeddings are those corresponding to subtrees containing all descendants - this is the notion of subtree most relevant to computer science and linguistics.

The subcategory of generic tree maps is opposite to the category of trees studied by Leinster [14, §7.3]. On Leinster's side of the duality, tree maps can be described in terms of set maps between the sets of nodes. On our side of the duality, tree maps are described in terms of set maps between the sets of edges. The category of generic injections is roughly the opposite of the category of trees studied ${ }^{1}$ by Ginzburg and Kapranov in their seminal paper [7]; the difference is that they exclude all trees with nullary nodes. In fact, most of the time they also exclude trees with unary nodes, and call the remaining trees reduced.

The subcategory $\mathbf{T E m} \boldsymbol{b}$, orthogonal to the generics, does not seem to have been studied before. It allows grafting of trees to be expressed as a pushout (1.1.19) and it carries the Grothendieck topology in terms of which the Segal condition is expressed (2.3.3).

0.0.2. Overview of Part 2: polynomial endofunctors in terms of trees. In the second part we describe polynomial endofunctors and monads as structures built from trees. Let $\boldsymbol{t} E \mathbf{m} \boldsymbol{b}$ and tree denote chosen skeleta of $\mathbf{T E m b}$ and Tree, respectively. Since $\boldsymbol{t} E \mathbf{m} \boldsymbol{b}$ is a subcategory in PolyEnd, there is a natural nerve functor PolyEnd $\rightarrow \operatorname{PrSh}(\boldsymbol{t E m b})$, and similarly there is a nerve functor PolyMnd $\rightarrow \operatorname{PrSh}($ tree $)$. These nerve functors are fully faithful, and we characterise their images. A main tool for these results is the theory of monads with arities due to Weber [21], which is reviewed in Section 2.0. Nerves of polynomial functors constitute an interesting application of Weber's theory, of a somewhat different flavour than the previously known examples, the new twist being that PolyEnd is not a presheaf category.

A key observation is that although PolyEnd itself is not a presheaf category, every slice of it is a presheaf category. This result relies on a notion of element of a polynomial endofunctor, introduced in Section 2.1: the elements of a polynomial endofunctor are the maps into it from elementary trees, i.e. trees with at most one node. The elementary trees, forming the subcategory elTr, play the role of representables: we show (2.1.3) that the slice category PolyEnd / P is naturally equivalent to the presheaf category $\operatorname{PrSh}(\mathrm{el}(\mathrm{P}))$, and that each polynomial endofunctor $\mathrm{P}$ is the colimit of a diagram of shape el(P) (cf. 2.1.7).

In Section 2.2 we come to the notions of generic morphism and generic factorisation, key notions in Weber's theory. We show that every element of a polynomial monad factors as generic followed by free, and the object appearing in the middle of the factorisation is a tree (2.2.4). This is a main ingredient in the proof that the freemonad monad on PolyEnd is a local right adjoint (2.2.6). We then show that trees

\footnotetext{
${ }^{1}$ It seems that the category they study is not the same as the category they define: their definition 1.1.4 does not seem to exclude contraction of external edges. I mention this curiosity as an illustration of the subtlety of formalising arguments with trees.
} 
provide arities for the free-monad monad on PolyEnd (cf. 2.2.10). With these facts established, Weber's general nerve theorem (2.0.5) implies the following characterisation (2.2.12): a presheaf on tree is a polynomial monad if and only if its restriction to $t E m b$ is a polynomial endofunctor.

What is here called the special nerve theorem (2.0.3), first proved by Leinster [15] and subsumed in the theory of Weber [21], concerns the case of a local right adjoint cartesian monad on a presheaf category; it characterises nerves in terms of the Segal condition. The Segal condition is about requiring certain canonical cocones to be sent to limit cones, and can also be formulated as a sheaf condition for a Grothendieck topology. The Segal condition makes sense also in the present case, and amounts to a sheaf condition on $\boldsymbol{t} \boldsymbol{E} \mathbf{m} \boldsymbol{b}$ (2.3.3). It is shown that the nerve of a polynomial endofunctor is always a sheaf (2.3.4), and we have an equivalence of categories $\boldsymbol{S h}(\boldsymbol{t} E \mathbf{m b}) \simeq$ $\operatorname{PrSh}($ elTr). However, the Segal condition is not enough to characterise nerves of polynomial monads. The special nerve theorem does apply to slices, though, (they are presheaf categories): for a fixed polynomial monad $\mathrm{P}$, monads over $\mathrm{P}$ are characterised (2.3.6) as presheaves on tree/P satisfying the Segal condition.

In the absolute case, one more condition is needed for a nerve theorem: it amounts to characterising the polynomial endofunctors among the presheaves on elTr. Presheaves on elTr are precisely (coloured) collections. We show that a collection is (isomorphic to) the nerve of a polynomial endofunctor if and only if it is (isomorphic to) the symmetrisation of a nonsymmetric collection (2.4.5). (More precisely, the category of polynomial endofunctors is the Kleisli category for the symmetrisation monad for nonsymmetric collections). Another characterisation is also obtained: the polynomial endofunctors are the projective objects in Coll with respect to colour-preserving surjections (2.4.8). Such collections are called flat.

The final section contains a big diagram relating the various objects involved: polynomial endofunctors and monads (as well as their planar versions), collections and operads (as well as the nonsymmetric versions), and the adjunctions and nerve functors relating them. The nerve functor for polynomial monads factors through the category of coloured operads. Polynomial monads are characterised as those operads whose underlying collection is flat.

A note about generality. In this paper, for simplicity, we only consider finite trees, and correspondingly we always assume our polynomial functors to be finitary. This is the natural level of generality from the viewpoint of operad theory, and for the sake of giving a formal construction of the category of trees of Moerdijk and Weiss, which was the original motivation for this work. Most results and constructions should generalise to wellfounded trees (in the category of sets) and arbitrary polynomial functors. I believe large parts of the theory will also generalise to an arbitrary locally cartesian closed category $\mathscr{E}$. Many proofs can be reinterpreted in the internal language of $\mathscr{E}$, but there are also some that cannot (e.g. involving complements), and new approaches may be required. This general case, perhaps more interesting from the viewpoint of type theory, is left to another occasion.

The ideas and techniques of this paper are currently being developed in another 
direction (in joint work with André Joyal) to account also for graphs. We introduce a new formalism for Feynman graphs and prove nerve theorems for cyclic and modular operads [10].

\subsection{Polynomial functors}

0.1.1. Notation. Throughout we denote by 0 the empty set and by 1 the singleton. We use the symbols + and $\sum$ for disjoint union of sets (or categories).

We recall some facts about polynomial functors. For further details and many other aspects of this fascinating topic, the reader is referred to the manuscript in preparation Notes on Polynomial Functors [12].

0.1.2. Polynomial functors. A diagram of sets and set maps like this

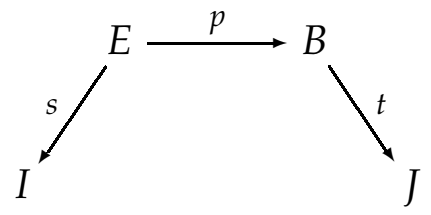

gives rise to a polynomial functor $\mathrm{P}:$ Set $/ I \rightarrow$ Set $/ J$ defined by

$$
\text { Set } / I \stackrel{s^{*}}{\longrightarrow} \operatorname{Set} / E \stackrel{p_{*}}{\longrightarrow} \operatorname{Set} / B \stackrel{t_{!}}{\longrightarrow} \operatorname{Set} / J .
$$

Here lowerstar and lowershriek denote, respectively, the right adjoint and the left adjoint of the pullback functor upperstar. In explicit terms, the functor is given by

$$
\begin{aligned}
\text { Set } / I & \longrightarrow \text { Set } / J \\
{[f: X \rightarrow I] } & \longmapsto \sum_{b \in B} \prod_{e \in E_{b}} X_{s(e)}
\end{aligned}
$$

where $E_{b}:=p^{-1}(b)$ and $X_{i}:=f^{-1}(i)$, and where the last set is considered to be over $J$ via $t_{\text {! }}$.

In this paper we shall only consider polynomial functors for which the map $p$ has finite fibres (equivalently, the functor preserves sequential colimits.) Such polynomial functors are called finitary. From now on, 'polynomial functor' means 'finitary polynomial functor'.

0.1.3. Categories of polynomial functors. (Cf. [6].) There is a category $\operatorname{Poly}(I, J)$ whose objects are the polynomial functors from Set/I to Set/J, and whose arrows are the cartesian natural transformations (i.e. natural transformations with cartesian naturality squares). A cartesian natural transformation $u: \mathrm{P}^{\prime} \Rightarrow \mathrm{P}$ between polynomial functors 
corresponds precisely to a commutative diagram

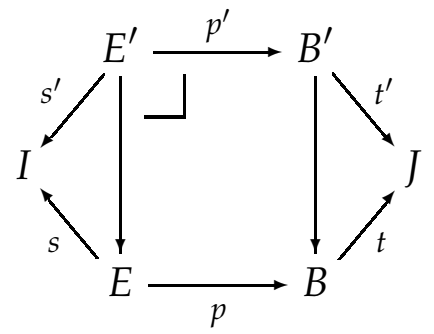

whose middle square is cartesian. In other words, giving $u$ amounts to giving a $J$-map $u: B^{\prime} \rightarrow B$ together with an $I$-bijection $E_{b^{\prime}}^{\prime} \stackrel{\sim}{\rightarrow} E_{u\left(b^{\prime}\right)}$ for each $b^{\prime} \in B^{\prime}$.

The composition of two polynomial functors is again polynomial [6]; this is a consequence of distributivity and the Beck-Chevalley conditions. Clearly the identity functor of Set/I is polynomial for each $I$. It follows that the categories $\operatorname{Poly}(I, J)$ form the hom categories of a 2-category Poly, which we see as a sub-2-category of Cat: the objects are the slice categories Set $/ I$, the arrows are the polynomial functors, and the 2-cells are the cartesian natural transformations. Since everything sits inside Cat, associativity of the compositions as well as the interchange law for composition of 2-cells are automatic.

From now on we shall only be concerned with the case $J=I$, i.e. the case of endofunctors. Throughout we use sans serif typeface for polynomial endofunctors, writing $\mathrm{P}=\left(P^{0}, P^{1}, P^{2}\right)$ for the functor represented by

$$
P^{0} \stackrel{s}{\longleftarrow} P^{2} \stackrel{p}{\longrightarrow} P^{1} \stackrel{t}{\longrightarrow} P^{0} .
$$

We shall use the letters $s, p, t$ for the three arrows in any diagram representing a polynomial endofunctor.

0.1.4. Polynomial monads. A polynomial monad is a monad in the 2-category Poly, i.e. a polynomial endofunctor $\mathrm{P}:$ Set $/ I \rightarrow$ Set /I with monoid structure in the monoidal category $($ PolyEnd $(I), \circ, \mathrm{Id})$. More explicitly still, there is specified a composition law $\mu: \mathrm{P} \circ \mathrm{P} \Rightarrow \mathrm{P}$ with unit $\eta: \mathrm{Id} \Rightarrow \mathrm{P}$, satisfying the usual associativity and unit conditions, and $\mu$ and $\eta$ are cartesian natural transformations. Let PolyMnd $(I)$ denote the category of polynomial monads on Set/I. The arrows are cartesian natural transformations respecting the monad structure.

0.1.5 Proposition. (cf. [5], [6].) The forgetful functor PolyMnd $(I) \rightarrow$ PolyEnd(I) has a left adjoint, denoted $\mathrm{P} \mapsto \overline{\mathrm{P}}$. The monad $\overline{\mathrm{P}}$ is the free monad on $\mathrm{P}$.

An explicit construction of $\bar{P}$ is given in 1.2.7.

0.1.6. Variable endpoints. It is necessary to consider also 2-cells between polynomial functors with different endpoints. Let PolyEnd denote the category whose objects are 
polynomial functors $\mathrm{P}=\left(P^{0} \leftarrow P^{2} \rightarrow P^{1} \rightarrow P^{0}\right)$ and whose morphisms are diagrams

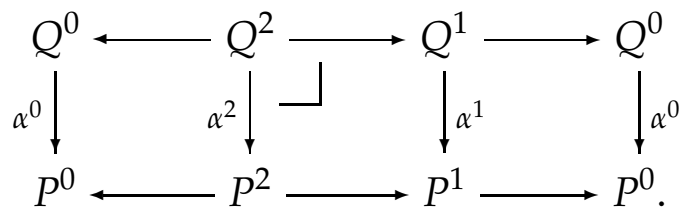

This category is fibred over Set by returning the endpoint [6].

A morphism $\alpha$ of polynomial functors is called injective, resp. surjective, if each of the three components, $\alpha^{0}, \alpha^{1}, \alpha^{2}$ is injective, resp. surjective.

Let PolyMnd denote the category whose objects are polynomial monads and whose morphisms are diagrams like (5), required to respect the monad structure. All we need to know about this is:

0.1.7 Proposition. (Cf. [6].) The forgetful functor PolyMnd $\rightarrow$ PolyEnd has a left adjoint $\mathrm{P} \mapsto \overline{\mathrm{P}}$, the free-monad functor. In other words, for each polynomial endofunctor $\mathrm{P}$ and each polynomial monad $\mathrm{M}$, there is a bijection

$$
\text { PolyEnd }(P, M) \leftrightarrow \text { PolyMnd }(\bar{P}, M),
$$

natural in $\mathrm{P}$ and $\mathrm{M}$.

This adjunction restricts to the adjunction of 0.1 .5 in each fibre. It is not a fibred adjunction, though.

0.1.8. Examples. The free-monoid monad

$$
\begin{aligned}
\mathrm{M}: \text { Set } & \longrightarrow \text { Set } \\
X & \longmapsto \sum_{n \in \mathbb{N}} X^{n}
\end{aligned}
$$

is polynomial: it is represented by the diagram

$$
1 \longleftarrow \mathbb{N}^{\prime} \longrightarrow \mathbb{N} \longrightarrow 1,
$$

where $\mathbb{N}^{\prime} \rightarrow \mathbb{N}$ is such that the fibre over $n$ has cardinality $n$, like for example $\mathbb{N}^{\prime}:=$ $\{(i, n) \in \mathbb{N} \times \mathbb{N} \mid i<n\}$ with the second projection. The slice category PolyMnd / M of polynomial monads over $\mathrm{M}$ is equivalent to the category of small multicategories (also called nonsymmetric coloured operads), and the fibre PolyMnd(1)/M corresponds to nonsymmetric operads.

The identity functor Id : Set $\rightarrow$ Set is clearly polynomial. The slice category PolyMnd/Id is equivalent to the category of small categories, and the fibre PolyMnd (1)/Id is equivalent to the category of monoids.

More generally, polynomial endofunctors over a polynomial monad T correspond to T-graphs, and polynomial monads over T correspond to small T-multicategories. All these results can be found in Leinster's book [14, §4.2], modulo the observation that any endofunctor with a cartesian natural transformation to a polynomial one is again polynomial, cf. [12]. 


\section{Trees in terms of polynomial endofunctors}

\subsection{Trees}

We shall define trees to be certain polynomial endofunctors. To motivate this definition, let us first informally explain what trees are supposed to be, and then show how to associate a polynomial endofunctor to a tree.

1.0.1. Trees. Our trees are non-planar finite rooted trees with boundary. Each node has a finite number of incoming edges and precisely one outgoing edge, always drawn below the node. The following drawings should suffice to exemplify trees, but beware that the planar aspect inherent in a drawing should be disregarded:

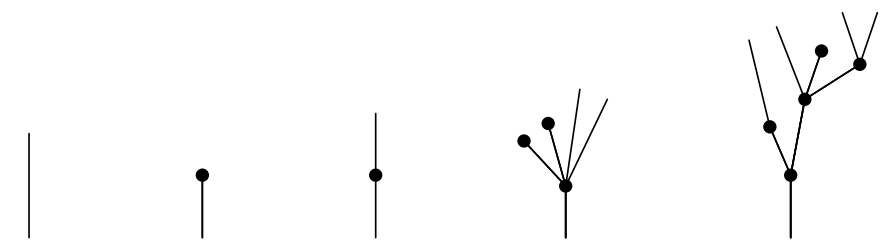

Note that certain edges (the leaves) do not start in a node and that one edge (the root edge) does not end in a node. The leaves and the root together form the boundary of the tree.

We shall give a formal definition of tree in a moment (1.0.3).

1.0.2. Polynomial functors from trees. Given a tree, define a polynomial functor

$$
T^{0} \stackrel{s}{\longleftarrow} T^{2} \stackrel{p}{\longrightarrow} T^{1} \stackrel{t}{\longrightarrow} T^{0},
$$

by letting $T^{0}$ be the set of edges, $T^{1}$ the set of nodes, and $T^{2}$ the set of nodes with a marked input edge, i.e. the set of pairs $(b, e)$ where $b$ is a node and $e$ is an incoming edge of $b$. The maps are the obvious ones: $s$ returns the marked edge of the node (i.e. $(b, e) \mapsto e$ ), the map $p$ forgets the mark (i.e. $(b, e) \mapsto b$ ), and $t$ returns the output edge of the node.

For example, the first three trees in the drawing above correspond to the following polynomial functors:

$$
1 \leftarrow 0 \rightarrow 0 \rightarrow 1 \quad 1 \leftarrow 0 \rightarrow 1 \rightarrow 1 \quad 2 \leftarrow 1 \rightarrow 1 \rightarrow 2 .
$$

The polynomial functors that arise from this construction are characterised by four simple conditions which are convenient to work with. We shall take this characterisation as our definition of tree:

1.0.3. Definition of tree. We define a finite rooted tree with boundary to be a polynomial endofunctor $\mathrm{T}=\left(T^{0}, T^{1}, T^{2}\right)$

$$
T^{0} \stackrel{s}{\longleftarrow} T^{2} \stackrel{p}{\longrightarrow} T^{1} \stackrel{t}{\longrightarrow} T^{0}
$$


satisfying the following four conditions:

(1) all the involved sets are finite.

(2) $t$ is injective

(3) $s$ is injective with singleton complement (called the root and denoted 1).

With $T^{0}=1+T^{2}$, define the walk-to-the-root function $\sigma: T^{0} \rightarrow T^{0}$ by $1 \mapsto 1$ and $e \mapsto t(p(e))$ for $e \in T^{2}$.

(4) $\forall x \in T^{0}: \exists k \in \mathbb{N}: \sigma^{k}(x)=1$.

The elements of $T^{0}$ are called edges. The elements of $T^{1}$ are called nodes. For $b \in T^{1}$, the edge $t(b)$ is called the output edge of the node. That $t$ is injective is just to say that each edge is the output edge of at most one node. For $b \in T^{1}$, the elements of the fibre $\left(T^{2}\right)_{b}:=p^{-1}(b)$ are called input edges of $b$. Hence the whole set $T^{2}=\sum_{b \in T^{1}}\left(T^{2}\right)_{b}$ can be thought of as the set of nodes-with-a-marked-input-edge, i.e. pairs $(b, e)$ where $b$ is a node and $e$ is an input edge of $b$. The map $s$ returns the marked edge. Condition (3) says that every edge is the input edge of a unique node, except the root edge. Condition (4) says that if you walk towards the root, in a finite number of steps you arrive there.

The edges not in the image of $t$ are called leaves. The root and the leaves together form the boundary of the tree.

From now on we just say tree for 'finite rooted tree with boundary'.

Let us briefly describe how to draw such a tree, i.e. give the converse of the construction in 1.0.2. Given $\left(T^{0}, T^{1}, T^{2}\right)$ we define a finite, oriented graph with boundary, i.e. edges are allowed to have loose ends: take the vertex set to be $T^{1}$ and the edge set to be $T^{0}$. The edges $x \in T^{0}$ which are not in the image of $t$ are the input edges of the graph in the sense that they do not start in a vertex. For each other edge $x$, we let it start in $b$ if and only if $t(b)=x$. (Precisely one such $b$ exists by axiom (2).) Clearly every $b$ occurs like this. Now we have decided where each edge starts. Let us decide where they end: the root edge 1 is defined to be the output edge of the graph, in the sense that it does not end in a vertex. For each other edge $e \neq 1$ (which we think of as $e \in T^{2}$ ), we let it end in $p(e)$. Note that the fibre of $p$ over a vertex $b$ consists of precisely the edges ending in $b$. Now we have described how all the edges and vertices are connected, and hence we have described a finite, oriented graph with boundary. Condition (4) implies that the graph is connected: every $e \neq 1$ has a 'next edge' $\sigma(e)$ distinct from itself, and in a finite number of steps comes down to the root edge. There can be no loops because there is precisely one edge coming out of each vertex, and linear cycles are excluded by connectedness and existence of a root. In conclusion, the graph we have drawn is a tree.

1.0.4. The trivial tree. The nodeless tree

$$
1 \longleftarrow 0 \longrightarrow 0 \longrightarrow 1 \text {, }
$$

(consisting solely of one edge) is called the trivial tree, and is denoted 1. 
1.0.5. One-node trees. For each finite set $E$ we have a one-node tree,

$$
E+1 \stackrel{s}{\longleftarrow} E \stackrel{p}{\longrightarrow} 1 \stackrel{t}{\longrightarrow} E+1,
$$

where $s$ and $t$ are the sum inclusions.

1.0.6. Elementary trees. An elementary tree is one with at most one node. That is, either a trivial tree or a one-node tree. These will play a fundamental role in the theory. We shall see in a moment that every tree is obtained by gluing together one-node trees along trivial trees in a specific way (grafting), while polynomial endofunctors are more general colimits of elementary trees.

1.0.7. Terminology. We define a partial order (called the tree order) on the edge set $T^{0}$ by declaring $x \leq y$ when $\exists k \in \mathbb{N}: \sigma^{k}(x)=y$. In this case $x$ is called a descendant of $y$, and $y$ is called an ancestor of $x$. In the particular case where $\sigma(x)=y$ and $x \neq y$, we say that $x$ is a child of $y$. If $\sigma(x)=\sigma(y)$ and $x \neq y$ we say that $x$ and $y$ are siblings. We define the distance from $x$ to $y$ to be $\min \left\{k \in \mathbb{N} \mid \sigma^{k}(x)=y\right\}$, whenever this set is nonempty. Note that the order induced on any 'up-set' is a linear order: if $e \leq x$ and $e \leq y$ then $x \leq y$ or $y \leq x$. The poset $T^{0}$ has a maximal element, namely the root; hence it has binary joins: the join of two edges is their nearest common ancestor. Every leaf is a minimal element for the tree order, but there may be other minimal elements. (Note that a partial order is induced on $T^{2} \subset T^{0}$, and also on $T^{1}$ (via $t$ ).)

\subsection{The category TEmb}

1.1.1. The category of trees and tree embeddings. Define the category TEmb to be the full subcategory of PolyEnd consisting of the trees. Hence a map of trees $\phi: S \rightarrow T$ is a diagram

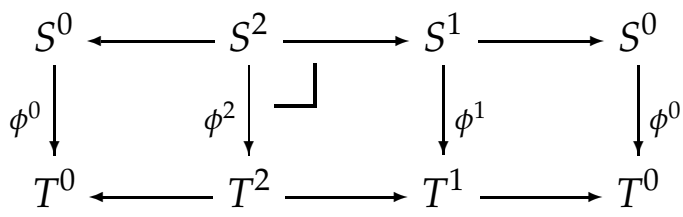

The cartesian condition amounts to 'arity preservation': the set of input edges of $b \in S^{1}$ maps bijectively onto the set of input edges of $\phi^{1}(b)$. Root and leaves are not in general preserved.

1.1.2 Lemma. Morphisms in TEmb preserve the childhood relation. That is, for a morphism $\phi: \mathrm{S} \rightarrow \mathrm{T}$, if $x$ is a child edge of $y$ in $\mathrm{S}$ then $\phi^{0}(x)$ is a child edge of $\phi^{0}(y)$ in $\mathrm{T}$. More generally, morphisms preserve distance.

Proof. To say that $x$ is a child of $y$ means that $x$ is not the root and $t(p(x))=y$. The property of not being the root is preserved by any map (cf. commutativity of the lefthand square in the diagram), so $\phi^{0}(x)$ is not the root either. Now apply $\phi$ and use that it commutes with $p$ and $t$, cf. (6). 


\subsubsection{Proposition. Every morphism in TEmb is injective.}

Proof. Let $\phi: \mathrm{S} \rightarrow \mathrm{T}$ in $\mathbf{T E m b}$. Let $r \in T^{0}$ denote the image of the root edge. Let $x, y$ be edges in $\mathrm{S}$ and suppose $\phi^{0}(x)=\phi^{0}(y)$. Since $\phi^{0}$ preserves distance we have $d(x, 1)=$ $d\left(\phi^{0}(x), r\right)=d\left(\phi^{0}(y), r\right)=d(y, 1)$. Since $x$ and $y$ have the same distance to the root, it makes sense to put $k:=\min \left\{n \in \mathbb{N} \mid \sigma^{n}(x)=\sigma^{n}(y)\right\}$, and $z:=\sigma^{k}(x)=\sigma^{k}(y)$ (nearest common ancestor). If $k>0$, then the edges $\sigma^{k-1}(x)$ and $\sigma^{k-1}(y)$ are both children of $z$, and by childhood preservation, we have $\phi\left(\sigma^{k-1}(x)\right)=\phi\left(\sigma^{k-1}(y)\right)$. But $\phi$ induces a bijection between the fibre $\left(S^{2}\right)_{z}$ and the fibre $\left(T^{2}\right)_{\phi^{0}(z)}$, so we conclude that already $\sigma^{k-1}(x)=\sigma^{k-1}(y)$, contradicting the minimality of $k$. Hence $k=0$, which is to say that already $x=y$. Hence we have shown that $\phi^{0}$ is injective. Since $t$ is always injective, it follows that also $\phi^{1}$ and $\phi^{2}$ are injective.

The proposition shows that the category $\mathbf{T E m b}$ is largely concerned with the combinatorics of subtrees, which we now pursue. It must be noted, though, that the category contains nontrivial automorphisms. In particular it is easy to see that

1.1.4 Lemma. The assignment of a one-node tree to every finite set as in 1.0.5 defines a fully faithful functor from the groupoid of finite sets and bijections into TEmb. (The essential image consists of the trees with precisely one node.)

1.1.5. Subtrees. A subtree of a tree $T$ is an isomorphism class of arrows $S \rightarrow T$ in $\mathbf{T E m b}$; more concretely it is an arrow $\mathrm{S} \rightarrow \mathrm{T}$ for which each of the three set maps are subset inclusions. Translating into classical viewpoints on trees, subtree means connected subgraph with the property that if a node is in the subgraph then all its incident edges are in the subgraph too.

Here are two examples:
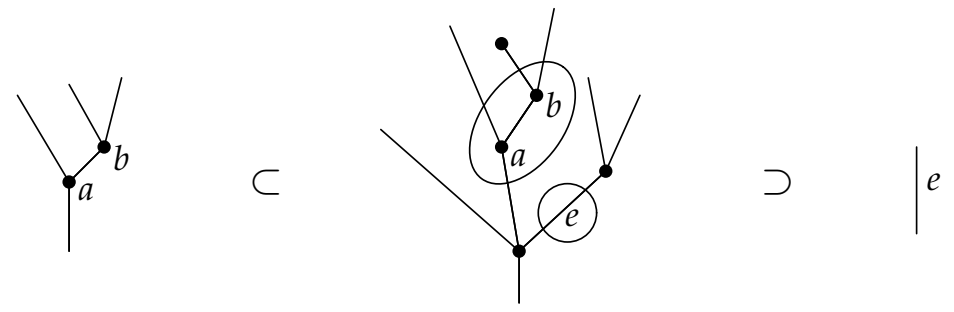

1.1.6. Edges. For each edge $x$ of $\mathrm{T}$ there is a subtree $\mathrm{I} \rightarrow \mathrm{T}$ given by

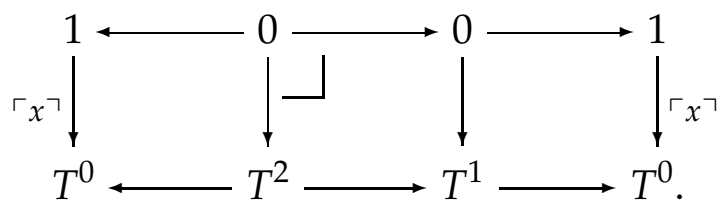

The subtree consists solely of the edge $x$. The edge is the root edge iff the left-hand square is a pullback, and the edge is a leaf iff the right-hand square is a pullback. 
1.1.7. One-node subtrees. For each node $b$ in $T$ there is a subtree inclusion

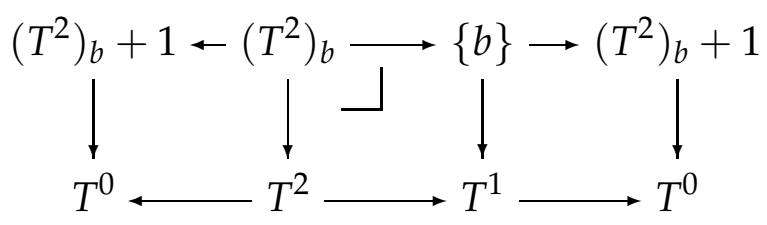

The vertical maps at the ends are the sum of $s \mid\left(T^{2}\right)_{b}$ and the map sending 1 to $t(b)$. The subtree defined is the local one-node subtree at $b$ : the node itself with all its incident edges.

1.1.8 Proposition. Let $\mathrm{R}$ and $\mathrm{S}$ be nontrivial subtrees in $\mathrm{T}$, and suppose that $R^{1} \subset S^{1}$. Then $\mathrm{R} \subset \mathrm{S}$. In particular, a nontrivial subtree is determined by its nodes.

Proof. We need to provide the dotted arrows in the diagram

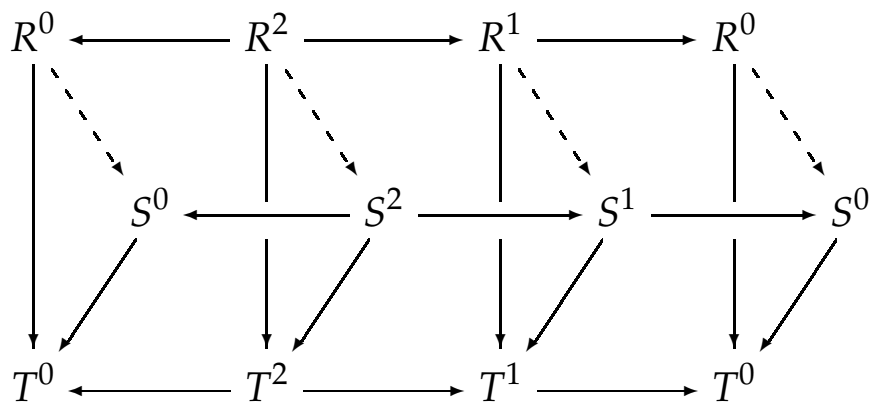

The arrow $R^{1} \rightarrow S^{1}$ is the assumed inclusion of nodes. For each node $b$ in $\mathrm{R}$ we have a bijection between the fibre $\left(R^{2}\right)_{b}$ and the fibre $\left(S^{2}\right)_{b}$. These bijections assemble into a map $R^{2} \rightarrow S^{2}$ and a cartesian square. Since $R^{0}=R^{2}+\{r\}$ where $r$ is the root edge of $\mathrm{R}$, to specify the arrow $R^{0} \rightarrow S^{0}$ it remains to observe that $r$ maps into $S^{0}$ : indeed, there is a $b \in R^{1}$ with $t(b)=r$. Hence $\phi^{0}(r)=\phi^{0}(t(b))=t\left(\phi^{1}(b)\right) \in S^{0}$.

1.1.9. Ideal subtrees. An ideal subtree is a subtree containing all the descendant nodes of its edges, and hence also all the descendant edges. (Hence it is a 'down-set' for the tree order (both with respect to nodes and with respect to edges), and just by being a subtree it is also closed under binary join.)

Each edge $z$ of a tree $T$ determines an ideal subtree denoted $D_{z}$ :

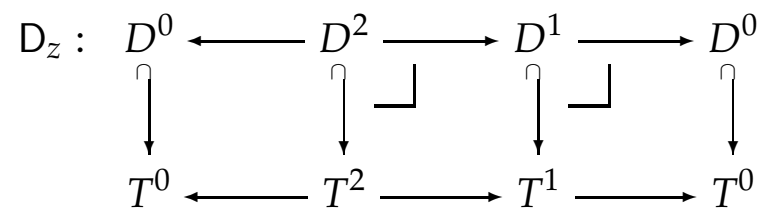

where

$$
\begin{aligned}
& D^{0}:=\left\{x \in T^{0} \mid x \leq z\right\}, \\
& D^{1}:=\left\{b \in T^{1} \mid t(b) \in D^{0}\right\}, \\
& D^{2}:=\left\{e \in T^{2} \mid t(p(e)) \in D^{0}\right\}=D^{0} \backslash\{z\} .
\end{aligned}
$$

It is easy to check that this is a tree; it looks like this: 


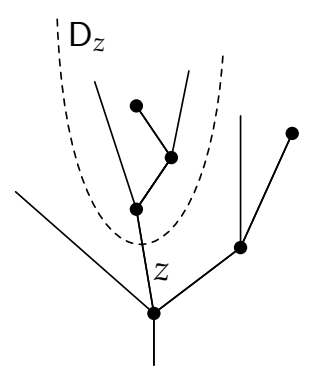

Note also that we have $x \in \mathrm{D}_{z} \Leftrightarrow x \leq z$.

1.1.10 Lemma. The following are equivalent for a tree embedding $\phi: \mathrm{S} \rightarrow \mathrm{T}$ :

1.The image subtree is an ideal subtree.

2.The right-hand square is cartesian (like in the above diagram).

3.The image of each leaf is again a leaf.

Proof. (1) $\Rightarrow(2)$ : clearly every ideal subtree $S \subset T$ is equal to $D_{z}$ for $z$ the root of $S$. Hence the embedding has cartesian right-hand square.

$(2) \Rightarrow(3)$ : a leaf in $S$ is characterised (1.1.6) as an edge for which the right-hand square is cartesian; composing with $\phi$ gives then again a cartesian right-hand square, so the edge is again a leaf in $T$.

(3) $\Rightarrow(1)$ : let $x$ be an edge in $S$, having a child node $b$ in T (that is, $p(b)=x$ ). This means $x$ is not a leaf in $\mathrm{T}$, and hence by assumption, not a leaf in S either. So $b$ is also in S.

1.1.11. Pruning. Using complements, it is not difficult to see that an edge $z \in T^{0}$ defines also another subtree which has the original root, but where all descendants of $z$ have been pruned. In other words, the ideal subtree $D_{z}$ is thrown away (except for the edge $z$ itself). Formally, with the notation of the ideal subtree: put $C^{1}:=T^{1} \backslash D^{1}$ and $C^{2}:=T^{2} \backslash D^{2}$. Then clearly we have a cartesian square

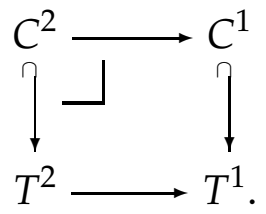

Now simply put $C^{0}:=C^{2}+\{1\}$ (the original root). It remains to see that the map $t: T^{1} \rightarrow T^{0}$ restricts to $C^{1} \rightarrow C^{0}$, but this follows from the fact that if $t(b)$ is not in $D^{0}$, then it must be in either $C^{2}$ or 1 . Using simple set theory, one readily checks that this is a tree again.

In any poset, we say that two elements $e$ and $e^{\prime}$ are incomparable if neither $e \leq e^{\prime}$ nor $e^{\prime} \leq e$. If two subtrees have incomparable roots then they are disjoint. Indeed, suppose the subtrees $\mathrm{S}$ and $\mathrm{S}^{\prime}$ of $\mathrm{T}$ have an edge $x$ in common. Then the totally ordered set of ancestors of $x$ in T will contain both the root of $S$ and the root of $S^{\prime}$, hence they are comparable. Clearly siblings are incomparable. In particular, if two subtrees have sibling roots, then they are disjoint. 
1.1.12 Lemma. Let $x$ and $y$ be edges of a tree T. Then the following are equivalent:

1. The ideal subtrees $\mathrm{D}_{x}$ and $\mathrm{D}_{y}$ are disjoint.

2. $x$ and $y$ are incomparable (i.e. neither $x \leq y$ nor $y \leq x$ ).

3. There exists a subtree in which $x$ and $y$ are leaves.

Proof. If $x \leq y$ then clearly $\mathrm{D}_{x} \subset \mathrm{D}_{y}$. On the other hand if $\mathrm{D}_{x}$ and $\mathrm{D}_{y}$ have an edge $e$ in common, then $e \leq x$ and $e \leq y$, and hence $x \leq y$ or $y \leq x$. Concerning condition (3): if $x$ and $y$ are leaves of a subtree, in particular they are both minimal, and in particular they are incomparable. Conversely, if they are incomparable, then we already know that the ideal subtrees they generate are disjoint, so we can prune at $x$ and $y$ to get a subtree in which $x$ and $y$ are leaves.

1.1.13. Root-preserving embeddings. An arrow $S \rightarrow T$ in $\mathbf{T E m b}$ is called root preserving if the root is mapped to the root. In other words, $\mathrm{S}$ viewed as a subtree of $T$ contains the root edge of $\mathrm{T}$ :

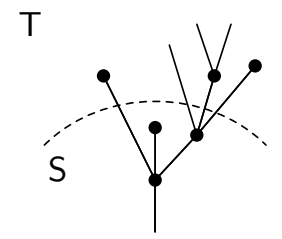

The root preserving subtrees are those that are up-sets in the tree order. It is easy to check that $S \rightarrow T$ is root-preserving if and only if the left-hand square is a pullback.

1.1.14 Lemma. If a tree embedding is both root preserving and ideal, then it is invertible (i.e. its image is the whole tree).

Proof. Indeed, if it is root preserving then its image contains 1 , and because it is ideal its image contains all other edges, as they are descendants of the root.

1.1.15 Proposition. Every arrow $\phi: S \rightarrow T$ in $\mathbf{T E m b}$ factors uniquely as a root-preserving map followed by an ideal embedding.

Proof. Put $r:=\phi^{0}$ (root), and consider the ideal subtree $\mathrm{D}_{r} \subset \mathrm{T}$. Since the map preserves the childhood relation, it is clear that all edges in $\mathrm{S}$ map into $\mathrm{D}_{r}$, and this map is root preserving by construction.

1.1.16. Remark. One can equally well factor every map the other way around: first an ideal embedding and then a root-preserving embedding. We will not have any use of that factorisation, though.

1.1.17 Lemma. A subtree is determined by its boundary. 
Proof. Let $\mathrm{S} \subset \mathrm{T}$ and $\mathrm{S}^{\prime} \subset \mathrm{T}$ be subtrees with common boundary. Suppose $b$ is a node of $\mathrm{S}$ which is not in $\mathrm{S}^{\prime}$. Since $b$ is in $\mathrm{S}$, for some $k$ we have $\sigma^{k}(t(b))=\operatorname{root}(\mathrm{S})=\operatorname{root}\left(\mathrm{S}^{\prime}\right)$. In this chain of nodes and edges, there is a node $b$ which is in $S$ but not in $S^{\prime}$, and such that $t(b)$ is an edge in $S^{\prime}$. This means $t(b)$ is a leaf in $S^{\prime}$ and hence a leaf in $S$, but this in turn implies that $b$ is not in $\mathrm{S}$, in contradiction with the initial assumption. So the two subtrees contain the same nodes. If they do contain nodes at all then they are equal by Lemma 1.1.8. If both subtrees are trivial, then they must coincide because their roots coincide.

1.1.18. Pushouts in PolyEnd. A polynomial functor P is a diagram in Set of shape

While pointwise sums are also sums in PolyEnd, pointwise pushouts are not in general pushouts in PolyEnd, because of the condition on arrows that the middle square be cartesian. Only pushouts over polynomial functors of shape ? $\leftarrow 0 \rightarrow 0 \rightarrow$ ? can be computed pointwise. In particular we can take pushouts over the trivial tree $1: 1 \leftarrow$ $0 \rightarrow 0 \rightarrow 1$. The pushout of the morphisms $\mathrm{S} \leftarrow 1 \rightarrow \mathrm{T}$ is the polynomial endofunctor given by

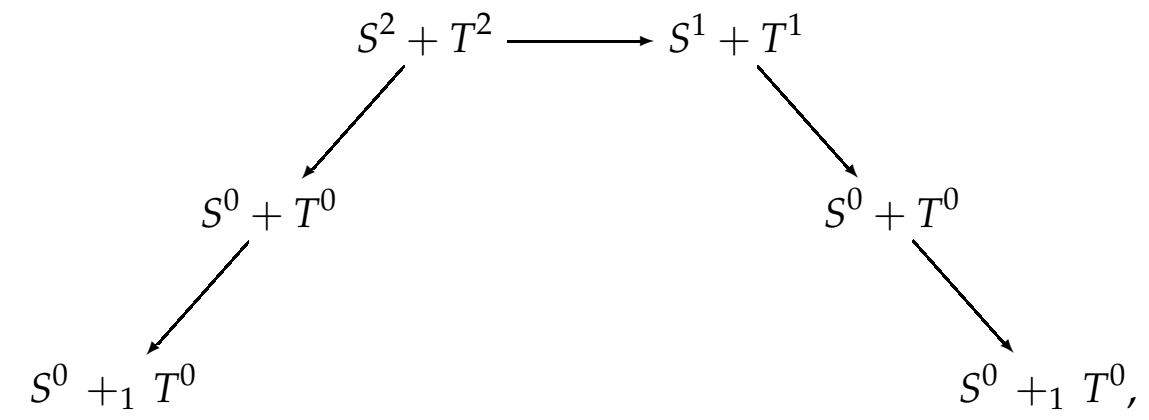

where $S^{0}+{ }_{1} T^{0}$ denotes the amalgamated sum over the singleton.

1.1.19 Proposition. Given a diagram of trees and tree embeddings

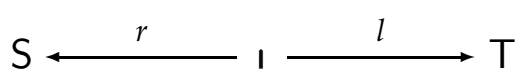

such that $r$ is the root edge in $\mathrm{S}$, and $\mathrm{l}$ is a leaf in $\mathrm{T}$, the pushout in PolyEnd is again a tree, called the grafting of $\mathrm{S}$ onto the leaf of $\mathrm{T}$, and denoted $\mathrm{S}+, \mathrm{T}$.

Proof. We check that the polynomial endofunctor (7) is a tree by inspection of the four axioms. Axiom 1: it is obvious the involved sets are finite. Axiom 2: we check that the right-hand leg is injective: to say that $l$ is a leaf of $T$ means it is not in the image of $t: T^{1} \rightarrow T^{0}$. So we can write $S^{1}+T^{1}=S^{1}+_{\{l\}}\left(\{l\}+T^{1}\right)$, and the map we want to prove injective is just the inclusion $S^{1}+T^{1}=S^{1}+\{l\}\left(\{l\}+T^{1}\right) \hookrightarrow S^{1}+\{l\} T^{0}$. Axiom 3: we check that the left-hand leg is injective and has singleton complement: this follows from the calculation $S^{0}+{ }_{1} T^{0}=\left(S^{2}+\{r\}\right)+{ }_{\{r\}} T^{0}=S^{2}+T^{0}=S^{2}+$ $T^{2}+1$ (where 1 denotes the root of the bottom tree $T$ ) Axiom 4: we check the walk-tothe-root condition: for $x \in S^{0}$, in a finite number of steps we arrive at $r=e=l$, and from here in another finite number of steps we come down to the root of $T$. 
1.1.20 Remark. More generally, the pushout of a root-preserving embedding along an ideal embedding is again a tree, and the two resulting maps are again root-preserving and ideal, respectively, as in this diagram

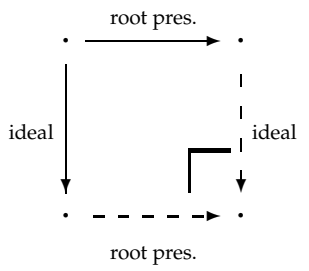

We will not need or prove this result here.

The following expresses the recursive characterisation of trees.

1.1.21 Proposition. A tree $T$ is either a nodeless tree, or it has a node $b \in T^{1}$ with $t(b)=1$; in this case for each $e \in\left(T^{2}\right)_{b}$ consider the ideal subtree $\mathrm{D}_{e}$ corresponding to $e$. Then the original tree $\mathrm{T}$ is the grafting of all the $\mathrm{D}_{e}$ onto the input edges of $b$.

Proof. The grafting exists by Proposition 1.1.19, and is a subtree in T by the universal property of the pushout. Clearly every node in $T$ is either $b$ or a node in one of the ideal subtrees, therefore the grafting is the whole tree, by Lemma 1.1.8.

1.1.22 Corollary. An automorphism of a tree amounts to permutation of siblings whose generated ideal subtrees are isomorphic.

Proof. Use the recursive characterisation of trees. By childhood preservation, an automorphism must send an edge $e$ to a sibling $e^{\prime}$. For the same reason it must map $\mathrm{D}_{e}$ isomorphically onto $\mathrm{D}_{e^{\prime}}$.

\subsubsection{Inner edges. An inner edge of a tree}

$$
T^{0} \stackrel{s}{\longleftarrow} T^{2} \stackrel{p}{\longrightarrow} T^{1} \stackrel{t}{\longrightarrow} T^{0}
$$

is one that is simultaneously in the image of $s$ and $t$. In other words, the set of inner edges is naturally identified with $T^{1} \times_{T^{0}} T^{2}$ considered as a subset of $T^{0}$; its elements are pairs $(b, e)$ such that $t(b)=s(e)$.

1.1.24 Corollary. Every nontrivial tree $\mathrm{T}$ is the grafting (indexed by the set of inner edges $\left.T^{1} \times_{T^{0}} T^{2}\right)$ of its one-node subtrees.

The elements of a tree $T$ are its nodes and edges. i.e. its elementary subtrees. These form a poset ordered by inclusion, and we denote this category el $(\mathrm{T})$. There is an obvious functor el $(T) \rightarrow T E m b$. This functor has a colimit which is just $T$. Indeed, each edge is included in at most two one-node subtrees of $\mathrm{T}$, and always as root in one and as leaf in the other; the colimit is obtained from these pushouts. The general notion of elements of a polynomial endofunctor will be introduced in Section 2.1. 


\subsection{P-trees and free monads}

The trees studied so far are in a precise sense abstract trees, whereas many trees found in the literature are structured trees, amounting to a morphism to a fixed polynomial functor. The structure most commonly found is planarity: a planar structure on a tree $\mathrm{T}$ is a linear ordering of the input edges of each node, i.e. a linear ordering on each fibre of $T^{2} \rightarrow T^{1}$. This amounts to giving a morphism $T \rightarrow \mathrm{M}$, where $\mathrm{M}$ is the free-monoid monad (0.1.8).

1.2.1. P-trees. Let $\mathrm{P}$ be a fixed polynomial endofunctor $P^{0} \leftarrow P^{2} \rightarrow P^{1} \rightarrow P^{0}$. By definition, the category of P-trees is the comma category $\mathbf{T E m b} / \mathrm{P}$ whose objects are trees $\mathrm{T}$ with a morphism $\mathrm{T} \rightarrow \mathrm{P}$ in PolyEnd. Explicitly, a P-tree is a tree $T^{0} \leftarrow T^{2} \rightarrow$ $T^{1} \rightarrow T^{0}$ together with a diagram

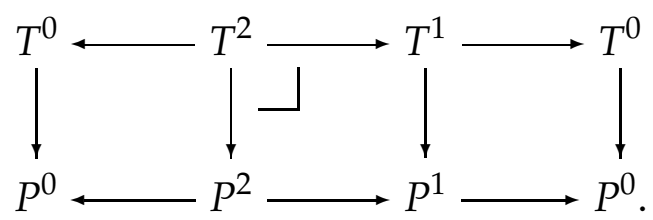

Unfolding further the definition, we see that a P-tree is a tree whose edges are decorated in $P^{0}$, whose nodes are decorated in $P^{1}$, and with the additional structure of a bijection for each node $n \in T^{1}$ (with decoration $b \in P^{1}$ ) between the set of input edges of $n$ and the fibre $\left(P^{2}\right)_{b}$, subject to the compatibility condition that such an edge $e \in\left(P^{2}\right)_{b}$ has decoration $s(e)$, and the output edge of $n$ has decoration $t(b)$. Note that the $P^{0}$-decoration of the edges is completely specified by the node decoration together with the compatibility requirement, except for the case of a nodeless tree. (The notion of $\mathrm{P}$-tree for a polynomial endofunctor $\mathrm{P}$ is closely related to the notion of $T_{S}$-tree of Baez and Dolan [1, Proof of Thm. 14], but they neglect to decorate the edge in the nodeless tree.)

If $\mathrm{P}$ is the identity monad, a $\mathrm{P}$-tree is just a linear tree. If $\mathrm{P}$ is the free-monoid monad, a P-tree is precisely a planar tree, as mentioned. If $\mathrm{P}$ is the free-nonsymmetric-operad monad on Set $/ \mathbb{N}$, the P-trees are the 3-dimensional opetopes, and so on: opetopes in arbitrary dimension are P-trees for a suitable P, cf. [1], [14, §7.1], [13].

1.2.2 Remark. It is important to note that P-trees are something genuinely different from just trees, in the sense that $\mathbf{T E m b}$ is not equivalent to $\mathbf{T E m b} / \mathrm{P}$ for any $P$. It is true of course that every tree admits a planar structure, i.e. a decoration by the freemonoid monad $\mathrm{M}$ (0.1.8): the possible diagrams

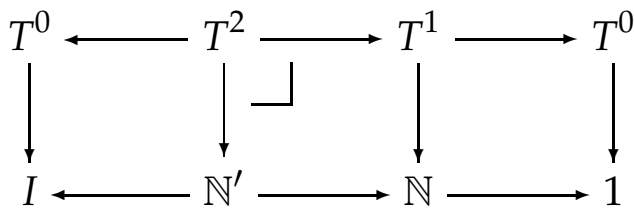

have to send a node $b \in T^{1}$ to its arity $n$ (the number of input edges), and then there are $n$ ! different choices for mapping the fibre to the $n$-element set $\mathbf{n}$, the fibre over $n$. 
The crucial property of P-trees is that they are rigid:

1.2.3 Proposition. P-trees have no nontrivial automorphisms.

Proof. Every automorphism of a tree consists in permuting siblings. Now in a P-tree, the set of siblings (some set $\left(T^{2}\right)_{b}$ ) is in specified bijection with $\left(P^{2}\right)_{\phi^{1}(b)}$, so no permutations are possible.

The basic results about trees, notably grafting (1.1.19) and the recursive characterisation (1.1.21), have obvious analogues for P-trees. We shall not repeat those results.

1.2.4. The set of $P$-trees. Let $P$ be a polynomial endofunctor. Denote by $\operatorname{tr}(P)$ the set of isomorphism classes of P-trees, i.e. isomorphism classes of diagrams

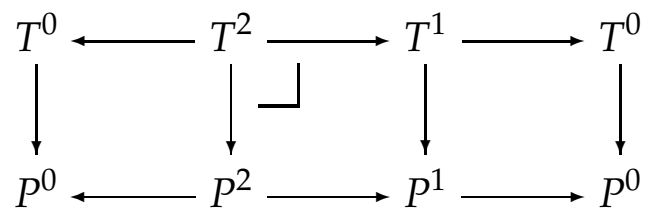

where the first row is a tree. Note that $\operatorname{tr}(\mathrm{P})$ is naturally a set over $P^{0}$ by returning the decoration of the root edge.

1.2.5 Theorem. If $\mathrm{P}$ is a polynomial endofunctor then $\operatorname{tr}(\mathrm{P})$ is a least fixpoint (i.e. initial Lambek algebra) for the endofunctor

$$
\begin{aligned}
1+\mathrm{P}: \text { Set } / P^{0} & \longrightarrow \text { Set } / P^{0} \\
X & \longmapsto P^{0}+P(X) .
\end{aligned}
$$

Proof. The proof uses the recursive characterisation of P-trees analogous to 1.1.21. For short, put $W:=\operatorname{tr}(\mathrm{P})$. We have

$$
\mathrm{P}(W)=\left\{(b, f) \mid b \in P^{1}, \quad P_{P^{0}}^{\left(P^{2}\right)_{b} \stackrel{f}{\longrightarrow} W}\right\}
$$

This set is in natural bijection with the set of P-trees with a root node decorated by $b \in P^{1}$. Indeed, given $(b, f) \in \mathrm{P}(W)$, we first consider the unique one-node $\mathrm{P}$-tree whose node is decorated by $b$. (This is well-defined: since $\left(P^{2}\right)_{b}$ is finite, the onenode tree is given as in 1.0.5, and the decorations are completely determined by the requirement that the node is decorated by $b$.) Now for each $e \in\left(P^{2}\right)_{b}$ we can graft the P-tree $f(e)$ onto the leaf $e$ of that one-node P-tree as in 1.1.19. The result is a P-tree D with root node decorated by $b$. Conversely, given a P-tree $\mathrm{D}$ with root node decorated by $b$, define $f:\left(P^{2}\right)_{b} \rightarrow W$ by sending $e$ to the ideal sub-P-tree $D_{e}$.

Now, $W$ is the sum of two sets: the nodeless $P$-trees (these are in bijection with $P^{0}$ ) and the P-trees with a root node. Hence we have $(1+P)(W) \stackrel{\sim}{\rightarrow} W$, saying that $W$ is a fixpoint. 
Finally, we must show that $W$ is a least fixpoint. Suppose $V \subset W$ is also a fixpoint. Let $W_{n} \subset W$ denote the set of P-trees with at most $n$ nodes. Clearly $W_{0} \subset V$. But if $W_{n} \subset V$ then also $W_{n+1} \subset V$ because each tree with $n+1$ nodes arises from some $(b, f)$ where $b$ decorates the root node and $f:\left(P^{2}\right)_{b} \rightarrow W_{n}$.

1.2.6. Historical remarks: wellfounded trees. Theorem 1.2 .5 has a long history: it is a classical observation (due to Lambek) that the elements of an initial algebra for an endofunctor $\mathrm{P}$ are tree-like structures, and that the branching profile of such trees depends on P. A very general version of the theorem is due to Moerdijk and Palmgren [16] providing categorical semantics for the notion of $W$-types (wellfounded trees) in Martin-Löf type theory. Briefly, under the Seely correspondence between (extensional) type theory and locally cartesian closed categories $\mathscr{E}$, the Sigma and Pi types correspond to dependent sums and products (as in (3)). The $W$ type constructor associates to a given combination $P$ of Sigma and Pi types a new inductive type $W_{P}$. Under the correspondence, $\mathrm{P}$ is a polynomial endofunctor on $\mathscr{E}$ (i.e. with $P^{0}$ terminal), and $W_{P}$ is its initial algebra.

The new feature of Theorem 1.2.5 (and the treatment leading to it) is to have trees and endofunctors on a common footing. This makes everything more transparent. Such a common footing was not possible in [16] because they only considered polynomial endofunctors $P$ with $P^{0}$ terminal. Trees cannot be captured by such, since it is essential to be able to distinguish the edges in a tree. The case of arbitrary polynomial functors was considered by Gambino and Hyland [5], corresponding to dependent type theory.

1.2.7. Construction of free monads. Let $\operatorname{tr}^{\prime}(\mathrm{P})$ denote the set of (isomorphism classes of) P-trees with a marked input leaf, i.e. the set of diagrams

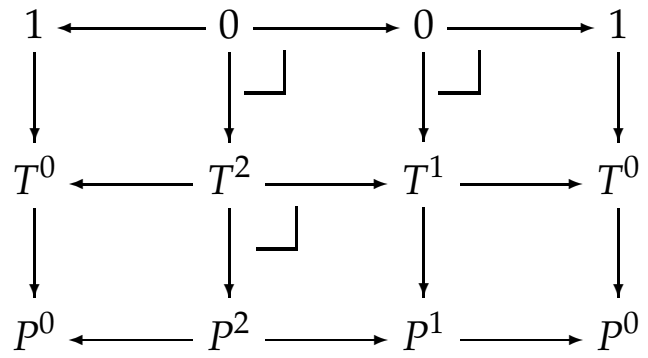

modulo isomorphism. (The cartesianness of the upper right-hand square says the edge is a leaf.) The set $\operatorname{tr}^{\prime}(\mathrm{P})$ is naturally an object of $\boldsymbol{S e t} / P^{0}$, the structure map $\operatorname{tr}^{\prime}(\mathrm{P}) \rightarrow P^{0}$ returning the decoration of the marked leaf. There is also the natural projection to $\operatorname{tr}(\mathrm{P})$ given by forgetting the mark. We get altogether a polynomial functor

$$
P^{0} \leftarrow \operatorname{tr}^{\prime}(\mathrm{P}) \rightarrow \operatorname{tr}(\mathrm{P}) \rightarrow P^{0}
$$

which we denote by $\bar{P}$. Its value on a set $X \rightarrow P^{0}$ is the set of $P$-trees with leaves decorated in $X$. More precisely, for a P-tree $S$, denote by $L_{S}$ the set of leaves of $S$, then

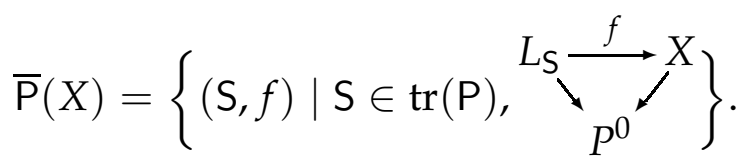


The polynomial functor $\bar{P}$ is naturally a monad: the multiplication map $\bar{P} \bar{P}(X) \rightarrow$ $\bar{P}(X)$ sends a P-tree $T$ with leaves decorated by other P-trees to the P-tree obtained by grafting the other $\mathrm{P}$-trees onto the leaves of $\mathrm{T}$. Note that the compatibility condition on the decorations states that the root edges of the decorating trees are precisely the leaves of the bottom tree, so the grafting makes sense. The unit for the multiplication is the map $P^{0} \rightarrow \overline{\mathrm{P}}\left(P^{0}\right)$ sending an edge $x$ to the trivial P-tree decorated by $x \in P^{0}$.

The construction $\mathrm{P} \mapsto \overline{\mathrm{P}}$ is clearly functorial. If $\alpha: \mathrm{Q} \rightarrow \mathrm{P}$ is a morphism of polynomial endofunctors, it is clear that $\bar{\alpha}^{1}: \operatorname{tr}(Q) \rightarrow \operatorname{tr}(P)$ sends trivial trees to trivial trees, and it is also clear it is compatible with grafting. Hence $\bar{\alpha}$ is a monad map.

1.2.8 Proposition. Let $\mathrm{P}$ be a polynomial endofunctor. The monad $\overline{\mathrm{P}}$ given by

$$
P^{0} \leftarrow \operatorname{tr}^{\prime}(P) \rightarrow \operatorname{tr}(P) \rightarrow P^{0}
$$

is the free monad on $\mathrm{P}$.

Proof. Given $X \rightarrow P^{0}$, put $W_{X}:=\overline{\mathrm{P}}(X)$, the set of P-trees with leaves decorated in $X$. In other words,

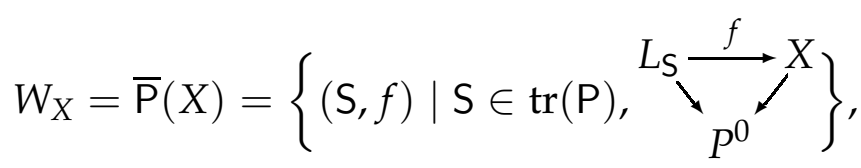

where $L_{S}$ denotes the set of leaves of a tree $S$. It follows from the argument of Lemma 1.2.5 that $W_{X}$ is a least fixpoint for the endofunctor $X+\mathrm{P}$, i.e. an initial object in $(X+$ $\mathrm{P})$-alg $\simeq X \downarrow P$-alg. Via the inclusion $\mathrm{P} \subset X+\mathrm{P}$ it also becomes a $\mathrm{P}$-algebra. The construction $X \mapsto W_{X}$ is clearly functorial and defines a functor

$$
\begin{aligned}
F: \text { Set } / P^{0} & \longrightarrow P \text {-alg } \\
X & \longmapsto W_{X} .
\end{aligned}
$$

To say that $W_{X}$ is initial in $(X+\mathrm{P})$-alg $\simeq X \downarrow P$-alg is equivalent to saying that $F$ is left adjoint to the forgetful functor $U: P$-alg $\rightarrow$ Set $/ P^{0}$, and therefore (e.g. by BarrWells [2, Theorem 4, p.311]), the generated monad $X \mapsto W_{X}$ is the free monad on $P$.

1.2.9. The free monad on a tree. We are particularly interested in the case where the polynomial endofunctor is itself a tree $T$. In this case we write $\operatorname{sub}(T)$ instead of $\operatorname{tr}(T)$, as we know that all maps between trees are injective. We restate this special case for emphasis:

1.2.10 Corollary. Let $\mathrm{T}$ be a tree. The monad $\overline{\mathrm{T}}$ given by

$$
T^{0} \leftarrow \operatorname{sub}^{\prime}(\mathrm{T}) \rightarrow \operatorname{sub}(\mathrm{T}) \rightarrow T^{0}
$$

is the free monad on $\mathrm{T}$. 


\subsection{The category Tree}

1.3.1. The category Tree. The category Tree is defined as the full subcategory of PolyMnd consisting of the free monads $\bar{T}$, where $T$ is a tree. This means taking the objects from TEmb and the morphisms from PolyMnd. More precisely the category Tree is given by the Gabriel factorisation (identity-on-objects/fully-faithful) of TEmb $\rightarrow$ PolyMnd:

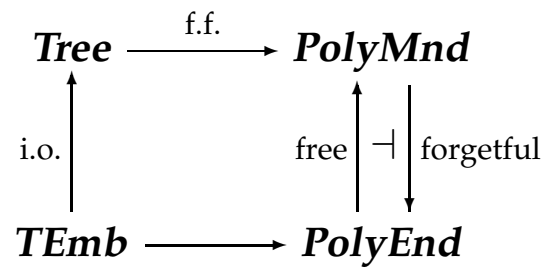

The category Tree is equivalent to the category $\Omega$ introduced by Moerdijk and Weiss [17], whose presheaves are called dendroidal sets. The category Tree is also described as the Kleisli category of the free-forgetful adjunction restricted to $\mathbf{T E m b}$. The arrows in the category Tree are by definition monad maps $\bar{S} \rightarrow \bar{T}$. By adjunction these correspond to maps of endofunctors $\mathrm{S} \rightarrow \overline{\mathrm{T}}$, and many properties of the category Tree can be extracted in this viewpoint, without ever giving an explicit description of the monad maps. However, remarkably, the following result holds:

1.3.2 Proposition. All maps of endofunctors $\overline{\mathrm{S}} \rightarrow \overline{\mathrm{T}}$ are monad maps. In other words, the forgetful functor Tree $\rightarrow$ PolyEnd is full.

This means that the maps in Tree have this surprisingly easy description: they are just commutative diagrams

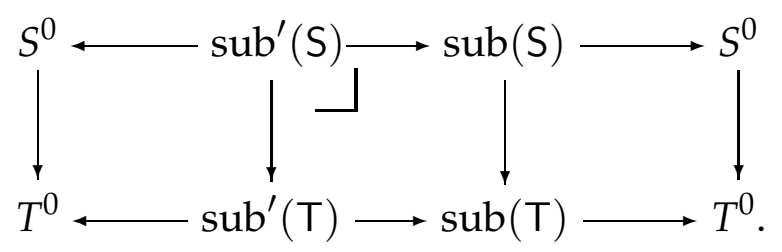

Proof of the Proposition. Since the monad structure is defined in terms of unit trees and grafting, the assertion follows from the following two lemmas which are of independent interest.

1.3.3 Lemma. Any map of polynomial endofunctors $\overline{\mathrm{S}} \rightarrow \overline{\mathrm{T}}$ maps trivial subtrees to trivial subtrees.

Proof. If $z$ is the root edge of a trivial subtree in S, then that trivial tree must map to a subtree of T with root $\phi(z)$, by commutativity of the right-hand square in (9). On the other hand, $z$ is also the unique leaf of that trivial tree, and by commutativity of the left-hand square in (9), the unique leaf of the image tree must be $\phi(z)$. Hence the image tree has the property that its root is equal to its unique leaf, hence it is trivial. 
1.3.4 Proposition. Every morphism $\phi: \overline{\mathrm{S}} \rightarrow \overline{\mathrm{T}}$ respects grafting. In other words, if a subtree $\mathrm{R} \in \operatorname{sub}(\mathrm{S})$ is a grafting $\mathrm{R}=\mathrm{A}+, \mathrm{B}$ then the image subtree $\phi^{1}(\mathrm{R}) \in \operatorname{sub}(\mathrm{T})$ is given by $\phi^{1}(\mathrm{R})=\phi^{1}(\mathrm{~A})+_{\phi^{1}(\mathrm{I})} \phi^{1}(\mathrm{~B})$.

Proof. The root of $A$ is I which is also a certain leaf of $B$. Hence the root of the image tree $\phi^{1}(A)$ is equal to $\phi^{1}(1)$ which is also a leaf of $\phi^{1}(B)$. Hence the grafting exists in T. It has root $\phi^{0}(\operatorname{root}(B))$ as required, and set of leaves $\phi^{0}($ leaves $(A)+$ leaves $(B) \backslash\{1\})$. So it has the same boundary as the image of $R$, so by Lemma 1.1.17 they agree.

1.3.5 Lemma. A map of polynomial endofunctors $\overline{\mathrm{S}} \rightarrow \overline{\mathrm{T}}$ is completely determined by its value on the edge set.

Proof. Let $\mathrm{R} \subset \mathrm{S}$ be an element of $\operatorname{sub}(\mathrm{S})$. The root of $\phi^{1}(\mathrm{R})$ must be the image of the root of $R$, by commutativity of the right-hand square of the representing diagram. Similarly, the set $L_{R}$ of leaves of $R$ is in bijection with the set of leaves of the image tree $\phi^{1}(\mathrm{R})$, by the cartesian condition on the middle square, but the latter set is also the image set $\phi\left(L_{\mathrm{R}}\right)$, by commutativity of the left-hand square. Hence the set of leaves of $\phi^{1}(R)$ are fixed, so altogether the boundary of $\phi^{1}(R)$ is completely determined, and we conclude by Lemma 1.1.17.

1.3.6 Corollary. If $\mathrm{S}$ is nontrivial, every map $\overline{\mathrm{S}} \rightarrow \overline{\mathrm{T}}$ is determined by its value on one-node subtrees. More precisely, the map is the grafting of maps on those one-node trees, indexed by the inner edges of S.

Proof. The first statement follows because the images of the nodes determine the images of their output and input edges, hence all edges have their image determined by the images of the nodes. For the more precise statement, note that the tree $\mathrm{S}$ is the grafting of its one-node trees indexed by its inner edges (cf. 1.1.24). The inner edges map to edges again, and since grafting is preserved, the whole map $\phi: \overline{\mathrm{S}} \rightarrow \overline{\mathrm{T}}$ is the grafting of the restrictions of $\phi$ to the one-node subtrees (indexed by the inner edges of S).

1.3.7 Proposition. Let $\phi$ be an arrow in Tree. Then $\phi^{0}$ preserves the tree order:

$$
x \leq y \quad \Rightarrow \quad \phi^{0}(x) \leq \phi^{0}(y) .
$$

Furthermore, if $x$ and $y$ are incomparable, then $\phi^{0}(x)$ and $\phi^{0}(y)$ are incomparable.

Proof. Suppose $x \leq y$. Let $S$ denote the minimal subtree with $y$ as root edge and $x$ as a leaf. Having $x$ as marked leaf makes $S$ an element in $\operatorname{sub}^{\prime}(T)$. By construction, $s(\mathrm{~S})=x$ and $t(p(\mathrm{~S}))=y$. Now apply $\phi$ and use the fact that $\phi$ commutes with each of the structure maps. Hence $\phi^{1}(\mathrm{~S})$ has $\phi^{0}(y)$ as root and $\phi^{0}(x)$ as marked leaf, and in particular $\phi^{0}(x) \leq \phi^{0}(y)$. For the second assertion, if $x$ and $y$ are incomparable, then by Lemma 1.1.12 there is a subtree in which $x$ and $y$ are leaves. Then $\phi^{0}(x)$ and $\phi^{0}(y)$ are leaves of the image subtree, and in particular incomparable.

Note that $\phi^{0}$ is not distance preserving, though, and that it is not necessarily injective. When it is injective it also reflects the tree order. 
1.3.8 Lemma. If $\phi: \overline{\mathrm{S}} \rightarrow \overline{\mathrm{T}}$ is a map of trees, then $\phi^{1}: \operatorname{sub}(\mathrm{S}) \rightarrow \operatorname{sub}(\mathrm{T})$ is inclusion preserving.

Proof. The statement is that if $\mathrm{Q} \subset \mathrm{R}$ are elements in $\operatorname{sub}(\mathrm{S})$ then we have $\phi^{1}(\mathrm{Q}) \subset$ $\phi^{1}(\mathrm{R})$ in $\operatorname{sub}(\mathrm{T})$. One way to see this is to observe that $\mathrm{Q}$ is determined by a subset of the nodes in R, cf. 1.1.8, and R is obtained from $Q$ by grafting those complementary one-node trees onto $Q$. By preservation of grafting (1.3.4), $\phi^{1}(R)$ is therefore obtained from $\phi^{1}(\mathrm{Q})$ by grafting certain subtrees onto it, and in particular $\phi^{1}(\mathrm{Q}) \subset \phi^{1}(\mathrm{R})$.

We have now gathered some basic knowledge of what general maps in Tree look like, and we already had a firm grip on the maps in TEmb. The following proposition summarises various characterisations of the maps in $\mathbf{T E m b}$ from the viewpoint of Tree, that is, it characterises the free maps:

1.3.9 Proposition. The following are equivalent for a map $\phi: \overline{\mathrm{S}} \rightarrow \overline{\mathrm{T}}$.

1. $\phi$ is free (i.e. of the form $\bar{\alpha}: \overline{\mathrm{S}} \rightarrow \overline{\mathrm{T}}$ ).

2. $\phi^{0}$ is distance preserving.

3. The image of a one-node subtree is a one-node subtree.

4. For every subtree $\mathrm{R} \subset \mathrm{S}$, the image subtree $\phi^{1}(\mathrm{R}) \subset \mathrm{T}$ is isomorphic to $\mathrm{R}$.

5. $\phi$ is injective, and if $\mathrm{R} \in \operatorname{sub}(\mathrm{T})$ is hit by $\phi^{1}$ then all the subtrees of $\mathrm{R}$ are hit too.

6. $\phi$ is injective, and if $\mathrm{R} \in \operatorname{sub}(\mathrm{T})$ is hit by $\phi^{1}$ then all edges of $\mathrm{R}$ are hit by $\phi^{0}$.

7. $\phi$ is injective, and all edges in $\phi^{1}(\mathrm{~S})$ are hit by $\phi^{0}$.

Proof. Straightforward verifications - omitted.

1.3.10 Corollary. In Tree, every isomorphism is free.

Another way to formulate Lemma 1.3.8 is that a map $\bar{S} \rightarrow \bar{T}$ restricts to any subtree $\mathrm{R} \subset \mathrm{S}$ to give a map $\overline{\mathrm{R}} \rightarrow \overline{\phi(\mathrm{R})}$. This is in fact a key observation, featured in the next proposition.

1.3.11. Boundary preserving maps. A map $\phi: \overline{\mathrm{S}} \rightarrow \overline{\mathrm{T}}$ is called boundary preserving if it takes the maximal subtree to the maximal subtree. Equivalently, it takes leaves to leaves (bijectively) and root to root. It is clear that the composite of two boundarypreserving maps is boundary preserving, and that every isomorphism is boundary preserving.

1.3.12 Lemma. Every surjection in Tree is boundary preserving.

Proof. If $\phi^{1}: \operatorname{sub}(\mathrm{S}) \rightarrow \operatorname{sub}(\mathrm{T})$ is surjective, in particular the maximal subtree $\mathrm{T} \in$ $\operatorname{sub}(\mathrm{T})$ is hit, and since $\phi^{1}$ is inclusion preserving by 1.3.8, T $\in \operatorname{sub}(\mathrm{T})$ must be hit by $\mathrm{S} \in \operatorname{sub}(\mathrm{S})$. 
1.3.13 Proposition. Every map of trees $\phi: \overline{\mathrm{S}} \rightarrow \overline{\mathrm{T}}$ factors essentially uniquely (i.e. uniquely up to unique isomorphism) as a boundary-preserving map followed by a free map. More precisely, the classes of boundary-preserving maps and free maps form an orthogonal factorisation system.

We shall see in 2.2.9 that the boundary-preserving maps are precisely the generic maps in the sense of Weber [21] (defined in 2.2.1 below). Generic maps are characterised by a universal property. The proposition states that Tree has generic factorisations, an important property for a Kleisli category.

Proof. The first statement will be a special case of Proposition 2.2.7, but here is the main argument: let $\mathrm{M}:=\phi^{1}(\mathrm{~S}) \in \operatorname{sub}(\mathrm{T})$ denote the image of the maximal subtree in $\mathrm{S}$, and let $\alpha: \mathrm{M} \rightarrow \mathrm{T}$ be the inclusion - this is a map in TEmb. Now $\bar{\alpha}: \overline{\mathrm{M}} \rightarrow \overline{\mathrm{T}}$ is the second factor in the wanted factorisation. Since $\phi^{1}$ is inclusion preserving by Lemma 1.3.8, we get also a map $\overline{\mathrm{S}} \rightarrow \overline{\mathrm{M}}$ which is boundary preserving by construction. It is easy to see that this factorisation is unique (up to a unique isomorphism). Finally, since both classes of maps contain the isomorphisms and are closed under composition, we have an orthogonal factorisation system.

1.3.14 Remark. There is a strong analogy between this boundary-preserving/free factorisation system in Tree and the root-preserving/ideal factorisation system in TEmb: in both cases the left-hand component is characterised in terms of a certain maxpreservation, while the right-hand component is characterised in terms of stability with respect to smaller elements, or equivalently in terms of preservation of certain minimal elements. Compare Lemma 1.1.10 with Proposition 1.3.9. We shall not pursue the analogy further.

We shall now describe the boundary-preserving maps in explicit terms. The main point of the analysis is Proposition 1.3.4 which says that any map out of a nontrivial tree is the grafting of its restriction to the one-node subtrees (also via Corollary 1.1.24). This is also just the content of the adjunction: to give a map $\bar{S} \rightarrow \overline{\mathrm{T}}$ is equivalent to giving $\mathrm{S} \rightarrow \overline{\mathrm{T}}$, so we just have to say where each node goes.

1.3.15. Boundary-preserving maps out of a one-node tree. Let $E$ denote a one-node tree with $n$ leaves, and suppose $\phi: \overline{\mathrm{E}} \rightarrow \overline{\mathrm{R}}$ is boundary preserving. By the cartesian condition, $\mathrm{R}$ necessarily has $n$ leaves, and for any such $\mathrm{R}$ there are precisely $n$ ! boundary-preserving maps from $\bar{E}$ to $\bar{R}$.

There are three cases to consider, depending on the number of nodes in $R$ : If $R$ has at least two nodes, then it has an inner edge, and since the map is boundary preserving this inner edge is not hit by $\phi^{0}$, so $\phi$ is not surjective. Since in $\mathrm{R}$ the root is different from any leaf, $\phi^{0}$ is injective, and therefore $\phi$ is injective. Here is a picture of such a node refinement: 


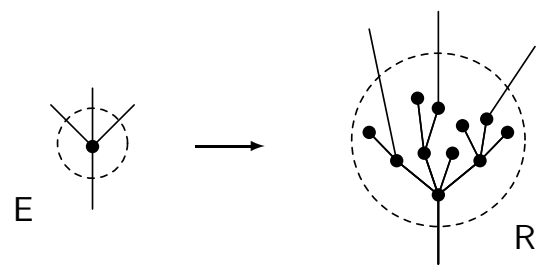

If $\mathrm{R}$ has precisely one node, clearly the map is an isomorphism. (This is not worth a picture.)

Finally there is a special case which occurs only for $n=1$ : then the tree $\mathrm{R}$ may be the trivial tree. In this case the two edges of $E$ are both sent to the unique edge of $R$, and the node is sent to the maximal subtree (also trivial) by the boundary-preservation assumption. In this case, $\phi$ is clearly surjective (and not injective). Here is a picture of such a unary-node deletion:

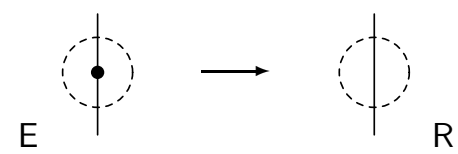

1.3.16. Boundary-preserving maps, general case. Consider now a general boundarypreserving map $\overline{\mathrm{S}} \rightarrow \overline{\mathrm{T}}$, and assume $\mathrm{S}$ is nontrivial. We know the map is the grafting of its restrictions to the one-node subtrees of $\mathrm{S}$. Let $\mathrm{E}$ be a one-node subtree of $\mathrm{S}$. We can factor the composite $\mathrm{E} \rightarrow \overline{\mathrm{S}} \rightarrow \overline{\mathrm{T}}$ into boundary-preserving followed by free:

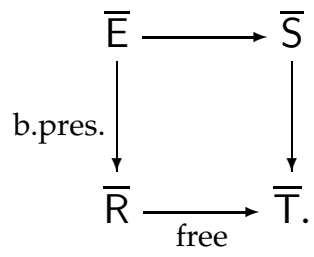

The subtree $R \in \operatorname{sub}(T)$ is the image of the subtree $E \in \operatorname{sub}(S)$. The map $\bar{E} \rightarrow \bar{R}$ is either a node refinement or a unary-node deletion or an isomorphism. The original map $\bar{S} \rightarrow \bar{T}$ is the grafting of all the maps $\bar{E} \rightarrow \bar{R}$ for $E$ running over the set of nodes in S. Here is a picture:

S

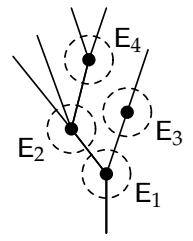

T

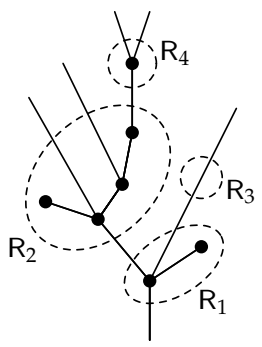

In conclusion, every boundary-preserving map $\bar{S} \rightarrow \bar{T}$ is the grafting of node refinements, unary-node deletions, and isomorphisms, indexed over the set of nodes in $\mathrm{S}$. It is clear that we can realise the grafting by refining (or deleting) the nodes one by 
one in any order, and in particular we can first do all the unary-node deletions (this amounts to a surjection), then all the node refinements (this amounts to an injection).

Since surjections are boundary-preserving (1.3.12), and since node refinements are not surjective we find:

1.3.17 Lemma. Every map in Tree factors essentially uniquely as a surjection followed by an injection. The surjections are generated by the unary-node deletions.

Combining the two factorisation systems we get a double factorisation system:

1.3.18 Proposition. Every morphism in Tree factors essentially uniquely as a surjection (a sequence of node deletions), followed by a boundary-preserving injection (a sequence of node refinements), followed by a free map (essentially a subtree inclusion).

1.3.19. Description of boundary-preserving injections into a given tree. To finish this first part of the paper, we show how to break the boundary-preserving injections into primitive maps. We already observed that we can refine one node at a time, but these are not the primitive maps. In order to characterise the primitive boundary-preserving injections, we change the viewpoint, looking at maps into a given tree instead of out of it:

Fix a tree $\mathrm{T}$, and suppose it has an inner edge $x=t(b)=s(e)$. We construct a new tree $\mathrm{T} / x$ by contracting the edge $x$, and exhibit a canonical boundary-preserving injection $\phi_{x}: \mathrm{T} / x \rightarrow \overline{\mathrm{T}}$ :

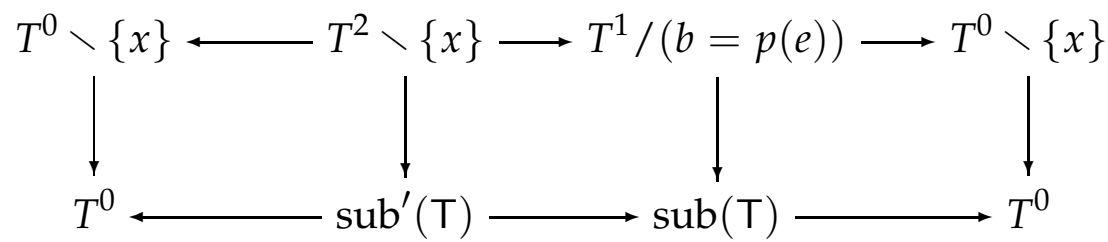

The maps are all obvious, except possibly $T^{1} /(b=p(e)) \rightarrow \operatorname{sub}(\mathrm{T})$ : this map sends the node $b=p(e)$ to the two-node subtree containing $b$ and $p(e)$, and sends all other nodes to their corresponding one-node subtree. It is clear that $\phi_{X}$ is boundary preserving and injective. The tree $T / x$ has one inner edge less than $T$. We can now repeat the process for any subset of the inner edges in $T$, and for each subset we get a different boundarypreserving injection into $\mathrm{T}$.

Conversely, every boundary-preserving injection $S \rightarrow T$ arises in this way. Indeed, we already know that these boundary-preserving injections are glued together from node refinements. The inner edges of the image trees form precisely the subset of edges we need to contract in order to recover the tree $\mathrm{S}$.

In conclusion, we have derived explicit descriptions for each of the four classes of maps. The surjections can be described more explicitly as deletion of unary nodes, and each surjection can be broken into a composite of maps deleting just one node. The boundary-preserving injections are described as node refinements, and each boundarypreserving injection can be broken into a sequence of 'primitive' refinements adding 
just one new node. The free maps are the 'arity-preserving' tree embeddings, which also can be given add-one-node wise. The new node is added either at a leaf (in which case the map is root preserving), or at the root (in which case the map is an ideal embedding).

1.3.20. Linear trees. A linear tree is one in which every node has precisely one input edge. The full subcategory of Tree consisting of the linear trees is equivalent to the simplex category $\Delta$. The factorisation systems restrict to $\Delta$, recovering the well-known fact that every map in $\Delta$ factors uniquely as a surjection followed by a top-and-bottompreserving injection, followed by distance-preserving injection. The primitive maps correspond to degeneracy and face maps in $\Delta$, which motivates the terminology employed by Moerdijk and Weiss [17]. They call the unary-node deletions degeneracy maps. The primitive node refinements they call inner face maps, and the primitive tree embeddings outer face maps. The inner face maps play a crucial role in their theory, to express horn-filling conditions for dendroidal sets [18].

\section{Polynomial endofunctors in terms of trees}

Since we are now going to consider presheaves, for size reasons we choose a skeleton for each of the categories $\mathbf{T E m b} \subset$ Tree, and denote them with a lowercase initial:

$$
t E m b \subset \text { tree. }
$$

Clearly these are small categories.

The embedding $i_{0}: \boldsymbol{t} \mathbf{E m} \boldsymbol{b} \rightarrow$ PolyEnd induces a nerve functor

$$
\begin{aligned}
N_{0}: \text { PolyEnd } & \longrightarrow \operatorname{PrSh}(\boldsymbol{t E m b}) \\
\mathrm{P} & \longmapsto \operatorname{Hom}_{\text {PolyEnd }}\left(i_{0}(-), \mathrm{P}\right) .
\end{aligned}
$$

Similarly, $i:$ tree $\rightarrow$ PolyMnd induces a nerve functor

$$
\begin{aligned}
N: \text { PolyMnd } & \longrightarrow \operatorname{PrSh}(\text { tree }) \\
\mathrm{P} & \longmapsto \operatorname{Hom}_{\text {PolyMnd }}(i(-), \mathrm{P})
\end{aligned}
$$

The goal of this second part is to characterise the image of these nerve functors.

\subsection{Background on nerve theorems}

We shall first recall the classical nerve theorem for categories, then review Weber's general framework for nerve theorems.

2.0.1. The nerve theorem for categories, after Berger [3]. It is classical that a simplicial set $X: \Delta^{\mathrm{op}} \rightarrow$ Set is (isomorphic to) the nerve of a small category if and only if the 'Segal condition' holds: for each $n \geq 1$, the natural map $X_{n} \rightarrow X_{1} \times X_{0} \cdots \times X_{0} X_{1}$ is 
an isomorphism. In the viewpoint of Berger and Weber, the starting point is the freecategory monad on $\mathbf{G r p h}=\boldsymbol{P r S h}(0 \rightrightarrows 1)$, the category of directed (non-reflexive) graphs. The free category on a graph $A$ has as objects the vertices of $A$ and as arrows the finite paths in $A$, i.e. graph maps from the finite, nonempty, linear graphs $[n]=$ $\{0 \rightarrow 1 \rightarrow \cdots \rightarrow n\}$ into $A$. Let $\Delta_{0} \subset$ Grph denote the full subcategory consisting of those linear graphs; note that all maps in $\Delta_{0}$ are injective. Equipped with the jointlysurjective topology, there is an equivalence of categories

$$
\operatorname{Sh}\left(\Delta_{0}\right) \simeq \text { Grph }
$$

(the topology is in fact determined by this equivalence).

Now $\Delta$ appears as the Kleisli category over $\Delta_{0}$ with respect to the free-category monad, and we have the diagram

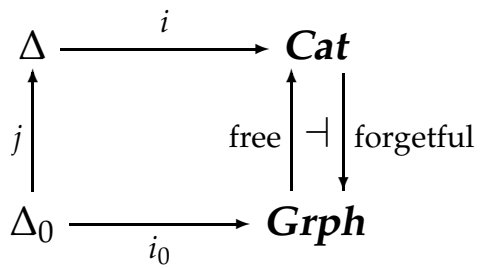

as in (8). The category $\Delta$ has generic-free factorisation; the generic maps are the end pointpreserving maps. (The free maps can be characterised as distance preserving.) The embeddings $i_{0}$ and $i$ are dense, hence induce fully faithful nerve functors

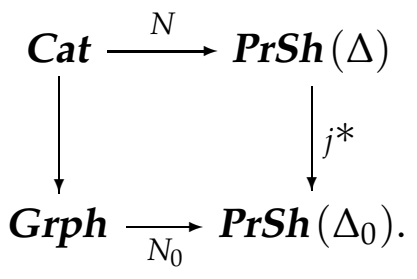

The classical nerve theorem can now be broken into two steps. The first says that $X: \Delta^{\text {op }} \rightarrow$ Set is in the essential image of $N$ if and only if $j^{*} X$ is in the essential image of $N_{0}$. The second step concerns presheaves on $\Delta_{0}$, and can be phrased in several equivalent ways: $X: \Delta_{0}^{\mathrm{op}} \rightarrow$ Set is a graph if and only if it is a sheaf (this is essentially a reformulation of the equivalence (11)). The sheaf condition amounts to the condition that certain cocones are sent to limit cones: each $[n] \in \Delta_{0}$ has a canonical expression as a colimit of copies of the two representables [0] and [1], and the condition amounts to saying that these cocones are sent to limit cones. This is just the usual Segal condition.

Berger [3] explained the nerve theorem for categories along these lines as a baby case of a similar theorem characterising strict $\omega$-categories as presheaves-with-Segalcondition on the cell category $\Theta$ of Joyal [9]. Leinster [15] proved a more general nerve theorem (cf. 2.0.3), and Weber [21] fitted everything into a natural (and more general) framework whose main notions we now recall. 
2.0.2. Local right adjoints and generic factorisation. (Cf. Weber [20].) A functor $F$ : $\mathscr{D} \rightarrow \mathscr{C}$ is called a local right adjoint if every slice

$$
\begin{aligned}
\mathscr{D} / X & \longrightarrow \mathscr{C} / F X \\
{[Y \rightarrow X] } & \longmapsto[F Y \rightarrow F X]
\end{aligned}
$$

is a right adjoint. (If $\mathscr{D}$ has a terminal object, the notion of local right adjoint coincides with Street's notion of parametric right adjoint [19].) This condition is equivalent to the following: every map in $\mathscr{C}$ of the form $a: A \rightarrow F X$ factors essentially uniquely as

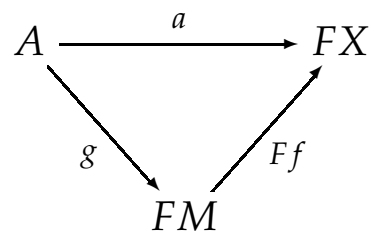

where $g: A \rightarrow F M$ is generic. We shall define generic in a moment (2.2.1).

Suppose now that $\mathscr{C}=\boldsymbol{P r S h}(\mathbb{C})$ and that $F$ is a local right adjoint cartesian monad on $\mathscr{C}$. In this case the local adjointness condition is equivalent to having generic factorisations just for maps of the form $C \rightarrow F 1$, where $C \in \mathbb{C}$ is a representable object in $\mathscr{C}$. Choose one such factorisation for each representable. Let $\Theta_{0}$ denote the full subcategory of $\mathscr{C}$ consisting of the objects $M$ appearing in these factorisations. Let $\Theta$ denote the Kleisli category of $F$ restricted to $\Theta_{0}$, i.e. the full subcategory of $F$-Alg given by the $F M$ for $M \in \Theta_{0}$. We have a diagram

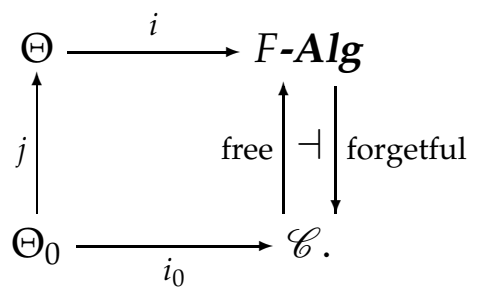

2.0.3. Special nerve theorem. (Cf. Leinster [15] and Weber [21].) The nerve functor $N: F-A l g \rightarrow \operatorname{PrSh}(\Theta)$ induced by $i$ is fully faithful, and its essential image consists of those presheaves on $\Theta$ satisfying the Segal condition, namely that the canonical cocones are sent to limit cones in Set.

2.0.4. Monads with arities. The conditions needed in order to get a nerve theorem have been further abstracted by Weber [21]. A monad with arities on a category $\mathscr{C}$ (not required to be a presheaf category or even to have a terminal object) consists of a monad $F$ (not required to be cartesian) and a full subcategory $i_{0}: \Theta_{0} \subset \mathscr{C}$ required to be dense and small, such that the following condition is satisfied: the left Kan extension

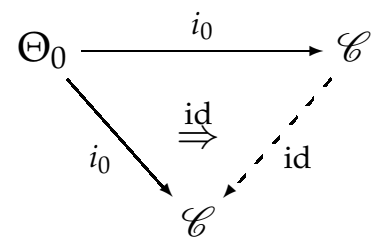


is preserved by the composite

$$
\mathscr{C} \stackrel{T}{\longrightarrow} \mathscr{C} \stackrel{N_{0}}{\longrightarrow} \operatorname{PrSh}\left(\Theta_{0}\right)
$$

(cf. the proof of Proposition 2.2.10 below). As above, denote by $\Theta$ the Kleisli category of $F$ restricted to $\Theta_{0}$, refer to Diagram (12), and let $N: F-\boldsymbol{A l g} \rightarrow \boldsymbol{P r S h}(\Theta)$ and $N_{0}$ : $\mathscr{C} \rightarrow \boldsymbol{P r S h}\left(\Theta_{0}\right)$ be the nerve functors induced by $i$ and $i_{0}$ respectively.

2.0.5. General nerve theorem. (Cf. Weber [21, Thm. 4.10].) If $\left(F, \Theta_{0}\right)$ is a monad with arities, then $N$ is fully faithful, and $X: \Theta^{\mathrm{op}} \rightarrow$ Set is in the essential image of $N$ if and only if its restriction to $\Theta_{0}$ is in the essential image of $N_{0}$.

The remainder of this article is concerned with establishing a nerve theorem for polynomial endofunctors and polynomial monads. In this case, the Segal condition is not enough to characterise the nerves, the reason being that PolyEnd is not a presheaf category: specifically it lacks a terminal object.

\subsection{Elements of a polynomial endofunctor}

Although PolyEnd is not a presheaf category, we shall see in a moment that all its slices are presheaf categories. The crucial construction is that of a category of elements of a polynomial endofunctor.

Recall that for an object $F$ of a presheaf category $\operatorname{PrSh}(\mathbb{C})$, the category of elements is the comma category $\mathbb{C} / F$, via the Yoneda embedding. Among the important properties of this construction is the fact that the composite functor

$$
\mathbb{C} / F \longrightarrow \mathbb{C} \stackrel{y}{\longrightarrow} \operatorname{PrSh}(\mathbb{C})
$$

has colimit F; it is called the canonical diagram for $F$. Second, there is a canonical equivalence of categories

$$
\boldsymbol{P r S h}(\mathbb{C}) / F \simeq \operatorname{PrSh}(\mathbb{C} / F) .
$$

We shall introduce the category of elements of a polynomial endofunctor $\mathrm{P}$, and establish the analogues of these two properties.

2.1.1. The category of elements of a polynomial endofunctor. To a given polynomial endofunctor $\mathrm{P}$, represented by $I \leftarrow E \rightarrow B \rightarrow I$, we associate a small category el(P), the category of elements of P. Since Poly is not a presheaf category, this notion is not the standard notion of category of elements. However, the terminology will be justified by Proposition 2.1.4 below. We first associate a bipartite graph to P: its vertex set is $I+B$ and every edge will go from an element in $I$ to an element in $B$. The set of edges is $B+E$. An edge $b \in B$ has source $t(b)$ and target $b$. An edge $e \in E$ has source $s(e)$ and target $p(e)$. Now define el $(\mathrm{P})$ to be the category generated by that bipartite graph; since the graph is bipartite, there are no composable arrows, so this step just 
amounts to adding an identity arrow for each object. It is clear that this construction is functorial, so we have defined a functor

$$
\text { el : PolyEnd } \rightarrow \text { Cat, }
$$

easily seen to be faithful.

2.1.2. Examples. Let $P$ be the identity functor (represented by $1 \leftarrow 1 \rightarrow 1 \rightarrow 1$ ). Then $\mathrm{el}(\mathrm{P})$ is a category with two objects and two parallel arrows (in addition to the identity arrows): $0 \Longrightarrow 1$. That is, the category whose presheaves are the graphs.

Let $\mathrm{P}$ be the free-monoid endofunctor $\mathrm{M}$ (represented by $1 \leftarrow \mathbb{N}^{\prime} \rightarrow \mathbb{N} \rightarrow 1$, cf. 0.1.8). Then el(M) has object set $1+\mathbb{N}=\{1,0,1,2, \ldots\}$, and its arrows all go from 1 to somewhere else. The set of arrows is $\mathbb{N}+\mathbb{N}^{\prime}$ (plus the identity arrows): for each $n \in \mathbb{N}$ there is an arrow $\mathrm{I} \rightarrow \mathrm{n}$, and for each $\{i<n\} \in \mathbb{N}^{\prime}$ there is another arrow $\mathrm{I} \rightarrow \mathrm{n}$. This is the category whose presheaves are the nonsymmetric coloured collections (2.4.4) (called multigraphs in [21], Example 2.14).

2.1.3 Proposition. There is an equivalence of categories

$$
\text { PolyEnd } / \mathrm{P} \simeq \operatorname{PrSh}(\mathrm{el}(\mathrm{P})) \text {. }
$$

Proof. To a given polynomial endofunctor Q over P:
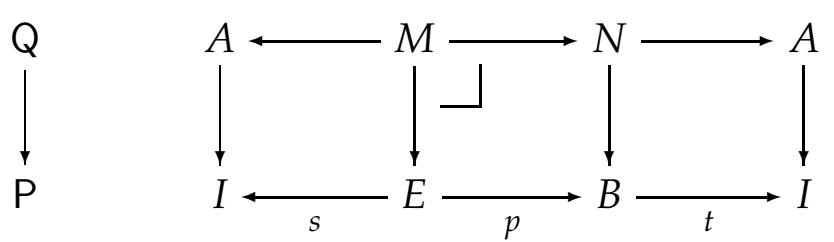

assign the presheaf $\widetilde{\mathrm{Q}}: \mathrm{el}(\mathrm{P})^{\mathrm{op}} \rightarrow$ Set whose value on the object $i$ is $A_{i}$, whose value on the object $b$ is $N_{b}$, whose value on the arrow $t(b) \rightarrow b$ is the restriction $N_{b} \rightarrow A_{t(b)}$, and whose value on the arrow $e: s(e) \rightarrow p(e)$ is the composite $N_{p(e)} \simeq M_{e} \rightarrow A_{s(e)}$.

Conversely, given a presheaf $X: \mathrm{el}(\mathrm{P})^{\text {op }} \rightarrow$ Set, we have in particular sets $X(i)$ or $X(b)$ for each of the objects $i$ or $b$ of $I+B$. Define a polynomial functor over $\mathrm{P}$ by

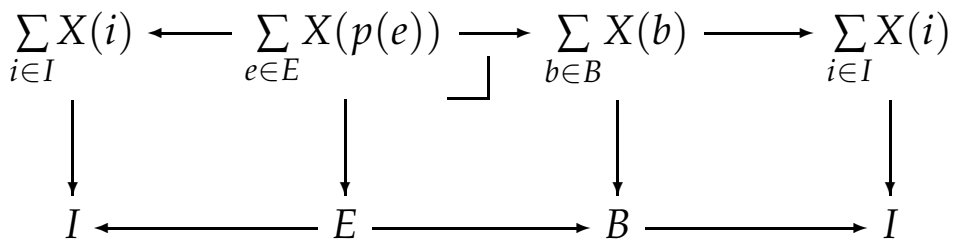

For each $b \in B$ there is an arrow $b: t(b) \rightarrow b$ in el(P) and hence a map $X(b) \rightarrow X(t(b))$, the sum of these maps constitute the endpoint component of the map. For each $e \in E$ there is an arrow $e: s(e) \rightarrow p(e)$ in el(P) and hence a map $X(p(e)) \rightarrow X(s(e))$; the sum of these maps constitute the left-hand map of the polynomial functor. The map in the middle is obvious.

It is easy to see that these assignments are functorial, and that the two constructions are inverse to each other. 
2.1.4 Proposition. Let $\mathrm{Q}$ be a polynomial endofunctor over $\mathrm{P}$, and let $\widetilde{\mathrm{Q}}: \operatorname{el}(\mathrm{P})^{\mathrm{op}} \rightarrow$ Set denote the corresponding presheaf via Proposition 2.1.3. Then there is a natural isomorphism between $\mathrm{el}(\mathrm{Q})$ and the category of elements of $\widetilde{\mathrm{Q}}$.

Proof. (We work just with the graphs, omitting the verification needed for identity arrows.) Let $\mathrm{Q} \rightarrow \mathrm{P}$ be given by

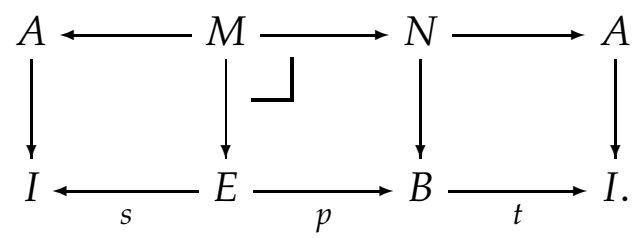

The elements of the presheaf $\widetilde{\mathrm{Q}}: \mathrm{el}(\mathrm{P})^{\mathrm{op}} \rightarrow$ Set are pairs $(u, x)$ where $u \in \operatorname{obj}(\mathrm{el}(\mathrm{P}))=$ $I+B$ and $x \in \widetilde{\mathrm{Q}}(u)$. In other words, an element of $\widetilde{\mathrm{Q}}$ is either a pair $(i, a)$, with $i \in I$ and $a \in \widetilde{\mathrm{Q}}(i)=A_{i}$, or a pair $(b, n)$, with $b \in B$ and $n \in \widetilde{\mathrm{Q}}(b)=N_{b}$. In conclusion the object set is $A+N$, as desired.

An arrow from element $(u, x)$ to element $(v, y)$ is a pair $(f, y)$ where $f: u \rightarrow v$ belongs to $\operatorname{arr}(\mathrm{el}(\mathrm{P}))=B+E$, and $y \in \widetilde{\mathrm{Q}}(v)$. In other words, an arrow is either a pair $(b, n)$ where $b: t(b) \rightarrow b$ in el $(\mathrm{P})$ and $n \in \widetilde{\mathrm{Q}}(b)=N_{b}$, or it is a pair $(e, m)$ where $e: s(e) \rightarrow p(e)$ in $\operatorname{el}(\mathrm{P})$ and $m \in \widetilde{\mathrm{Q}}(p(e))=N_{p(e)} \simeq M_{e}$. In conclusion, the set of arrows of the category of elements of $\widetilde{\mathrm{Q}}$ is $N+M$, and it is clear from the construction that their sources and targets are as required.

2.1.5. Elementary trees. Let EITr denote the full subcategory of TEmb consisting of the elementary trees, i.e. trees with at most one node. Let elTr denote a fixed skeleton of ElTr: we fix one trivial tree । and one one-node tree $\mathrm{n}$ for each $n \in \mathbb{N}$. There are $n+1$ arrows from 1 to $n$, one for each leaf and one for the root edge, and in addition to these arrows, each object $n$ has $n$ ! endomorphisms, all invertible. Henceforth we use the symbol $n$ to denote an arbitrary object of elTr, possibly the trivial tree.

For each polynomial endofunctor $\mathrm{P}$, there is a canonical equivalence of categories

$$
\mathrm{el}(\mathrm{P}) \simeq \operatorname{ElTr} / \mathrm{P}:
$$

to each element $i \in I$ corresponds the trivial P-tree

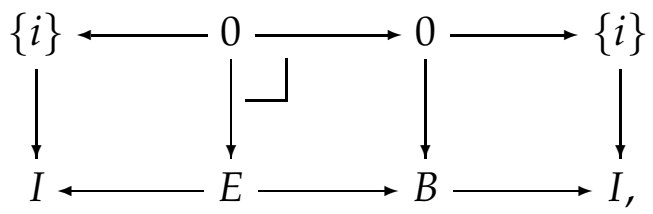

and to each element $b \in B$ corresponds the one-node P-tree

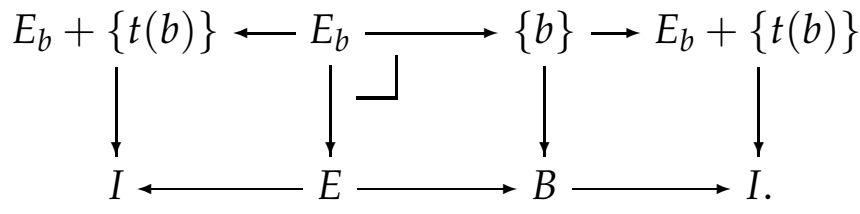


In particular, each element of $\mathrm{P}$ can be viewed as a morphism $\mathrm{n} \rightarrow \mathrm{P}$ (in analogy with the situation for presheaves), and we can think of ElTr as the category of representables. (We shall come back to the relation between PolyEnd and the presheaf category $\boldsymbol{P r S h}(\mathbf{e l T r})$ in Section 2.4.)

The equivalences we have established fit into this diagram (commutative up to isomorphism):

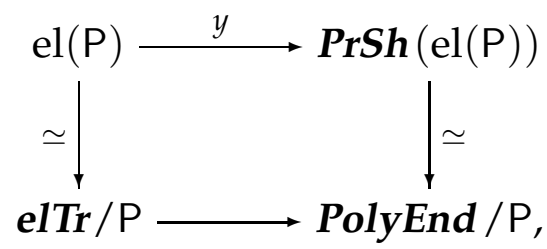

so locally elTr $\rightarrow$ PolyEnd is the Yoneda embedding of the representables.

2.1.6. The canonical diagram for $P$. The composite functor

$$
D_{\mathrm{P}}: \quad \mathrm{el}(\mathrm{P}) \longrightarrow \text { PolyEnd } / \mathrm{P} \longrightarrow \text { PolyEnd }
$$

is called the canonical diagram for $\mathrm{P}$, in view of the following result:

2.1.7 Proposition. The colimit of $D_{\mathrm{P}}$ is $\mathrm{P}$.

Proof. For $\mathrm{Q}$ over $\mathrm{P}$ we have

$$
\operatorname{colim}(\mathrm{el}(\mathrm{Q}) \rightarrow \text { PolyEnd } / \mathrm{P})=\mathrm{Q}
$$

since PolyEnd / P is a presheaf category by 2.1.3, and since the notion of elements is the usual one in this case by 2.1.4. The result now follows since the forgetful functor PolyEnd / P $\rightarrow$ PolyEnd preserves colimits. (It is a basic fact that PolyEnd has binary products, cf. [12], so $\mathrm{P} \times-$ is right adjoint to the forgetful functor in question.)

\subsection{Generic factorisation, and trees as arities}

The main results of this section are that the free-monad monad on PolyEnd is a local right adjoint, or equivalently that it has generic factorisations, and that trees are arities for the free-monad monad. We first show that elements of free monads have generic factorisation and that the resulting middle objects are precisely the trees. This is an explicit verification relying on our good handle on trees and free monads. The second step is to establish local right adjointness from the first result using the density of elementary trees. Finally we find that for trees the notions of generic and boundarypreserving coincide, as claimed in Section 1.3. From this it follows readily that trees satisfy Weber's axioms for arities.

2.2.1. Generic maps. The notion of generic map was introduced by Weber [20] generalising the notion of generic element of [8]. The following special case of the notion is 
the most useful. Let $F$ be a monad on a category $\mathscr{C}$. An arrow $g: A \rightarrow F B$ in $\mathscr{C}$ is called generic (with respect to $F$ ) if for every diagram

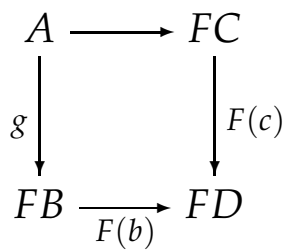

there is a unique arrow $u: B \rightarrow C$ such that

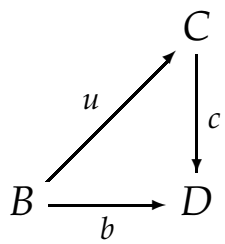

and

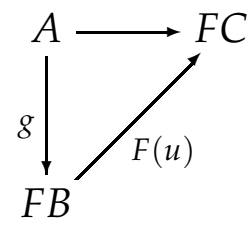

The endofunctor $F$ is said to admit generic factorisations if every $A \rightarrow F D$ admits a factorisation into a generic map followed by one in the image of $F$. (This condition is equivalent to being a local right adjoint.) Such a factorisation is then necessarily unique up to unique isomorphism. The maps of the form $A \rightarrow F D$ correspond by adjunction to $F$-algebra maps $F A \rightarrow F D$, which we shall also call generic. All the involved maps can then be seen as living in the Kleisli category of $F$. From this perspective, generic factorisations amount to having an orthogonal factorisation system generic/free in the Kleisli category. (To be correct, the right hand class of the factorisation system should be saturated with the isomorphisms. In our case all isomorphisms will already be free.)

2.2.2 Lemma. If $g: \overline{\mathrm{S}} \rightarrow \overline{\mathrm{T}}$ is a boundary-preserving map between trees, then $g$ is generic in PolyEnd (with respect to the free-monad monad $\mathrm{P} \mapsto \overline{\mathrm{P}}$ ).

Proof. Given a square

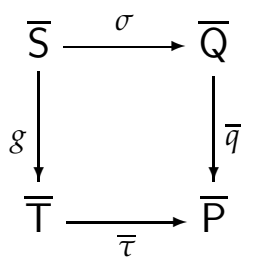

let $\mathrm{R} \rightarrow \mathrm{Q}$ denote the $\mathrm{Q}$-tree $\sigma^{1}(\mathrm{~S})$. It image under $\bar{q}^{1}$ is just $\mathrm{R} \rightarrow \mathrm{Q} \rightarrow \mathrm{P}$. Going the other way around the square, the maximal subtree $S$ is first mapped to $T \in \operatorname{sub}(T)$ (by boundary preservation), and then to the P-tree $\tau: T \rightarrow P$. Since the diagram commutes, $\mathrm{R}$ and $\mathrm{T}$ represent the same $\mathrm{P}$-tree, and since $\mathrm{P}$-trees have no nontrivial automorphisms, there is a unique isomorphism $T \stackrel{\sim}{\rightarrow} R$, and hence a unique diagonal filler $d: \mathrm{T} \rightarrow \mathrm{Q}$ for the square.

We shall see in a moment that the converse of the lemma is true as well. 
2.2.3. Generic factorisation of elements of free monads. The key point towards getting all generic factorisations is the following easy result:

2.2.4 Lemma. Every element $s: \mathrm{n} \rightarrow \overline{\mathrm{P}}$ of a free monad $\overline{\mathrm{P}}$ factors essentially uniquely as

$$
\overline{\mathrm{n}} \stackrel{g}{\longrightarrow} \overline{\mathrm{T}} \stackrel{\bar{f}}{\longrightarrow} \overline{\mathrm{P}},
$$

where the middle object $\mathrm{T}$ is a tree, $\mathrm{g}$ is boundary preserving (hence generic), and $\bar{f}$ is free.

Proof. If $\mathrm{n}$ is the trivial tree, the map $s: \mathrm{n} \rightarrow \overline{\mathrm{P}}$ singles out a single element $x \in P^{0}$, and $s$ is the free map

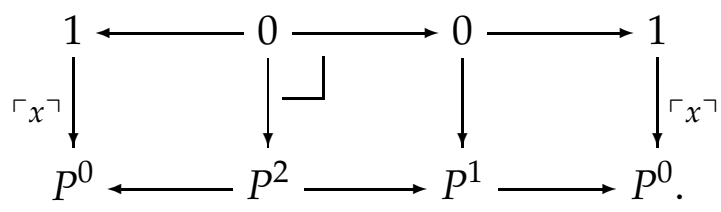

Hence we can take $T=n$, and $g$ is just the identity.

If $\mathrm{n}$ is a one-node tree, the unique element in $n^{1}$ maps to some element in $(\bar{P})^{1}=$ $\operatorname{tr}(P)$, i.e. a P-tree $f: \mathrm{T} \rightarrow \mathrm{P}$. The cartesian condition on maps ensures there is a bijection between the input edges of the unique node in $n$ and the leaves of $T$, hence a unique map $\overline{\mathrm{n}} \rightarrow \overline{\mathrm{T}}$ making the triangle commute - clearly this map is boundary preserving.

2.2.5. The spine. Fix a polynomial endofunctor $P$, and assume a choice of a generic factorisation for every element of $\bar{P}$, as in Lemma 2.2.4. These factorisations fit together to define a functor

$$
E_{\mathrm{P}}: \operatorname{el}(\overline{\mathrm{P}}) \rightarrow \text { PolyEnd / } \mathrm{P}
$$

(sometimes called the spine relative to $P$ ) sending an element of $\bar{P}$ to the tree appearing in the factorisation. Up to isomorphism, every P-tree $T \rightarrow P$ arises like this: just precompose $\mathrm{T} \rightarrow \mathrm{P}$ with a boundary-preserving map from the one-node tree with the same number of leaves as $T$. (Note that if $n$ is unary, its node may be mapped to a trivial P-tree. Hence the functor $E_{\mathrm{P}}$ is not injective on objects, not even on isomorphism classes of objects.)

2.2.6 Proposition. The monad $\mathrm{P} \mapsto \overline{\mathrm{P}}$ is a local right adjoint. That is, for each $\mathrm{P}$, the natural functor

$$
\begin{aligned}
\text { PolyEnd } / \mathrm{P} & \longrightarrow \text { PolyEnd } / \overline{\mathrm{P}} \\
{[\mathrm{Q} \rightarrow \mathrm{P}] } & \longmapsto[\overline{\mathrm{Q}} \rightarrow \overline{\mathrm{P}}]
\end{aligned}
$$

has a left adjoint. 
Proof. The asserted left adjoint will be the left Kan extension of $E_{\mathrm{P}}$ along the Yoneda embedding $y: \operatorname{el}(\overline{\mathrm{P}}) \rightarrow \boldsymbol{P r S h}(\mathrm{el}(\overline{\mathrm{P}})) \simeq$ PolyEnd $/ \overline{\mathrm{P}}$ :

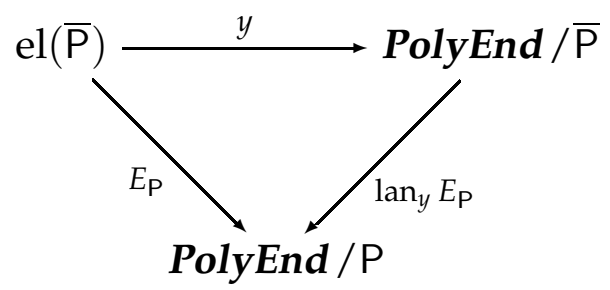

The functor $\operatorname{lan}_{y} E_{\mathrm{P}}$ sends a polynomial endofunctor $\mathrm{F} \rightarrow \overline{\mathrm{P}}$ to the colimit of the functor

$$
\mathrm{el}(\mathrm{F}) \longrightarrow \mathrm{el}(\overline{\mathrm{P}}) \stackrel{E_{\mathrm{P}}}{\longrightarrow} \text { PolyEnd } / \mathrm{P} \text {. }
$$

Using the identification PolyEnd $/ \overline{\mathrm{P}} \simeq \operatorname{PrSh}(\mathrm{el}(\overline{\mathrm{P}}))$, it is a general fact that $\operatorname{lan}_{y}\left(E_{\mathrm{P}}\right)$ has a right adjoint

$$
\begin{aligned}
\text { res : PolyEnd } / \mathrm{P} & \longrightarrow \operatorname{PrSh}(\mathrm{el}(\overline{\mathrm{P}})) \\
{[q: \mathrm{Q} \rightarrow \mathrm{P}] } & \longmapsto\left[[s: \mathrm{n} \rightarrow \overline{\mathrm{P}}] \mapsto \operatorname{Hom}_{\text {PolyEnd } / \mathrm{P}}\left(E_{\mathrm{P}}(s), q\right)\right] .
\end{aligned}
$$

So to establish the claim we must show that res is isomorphic to (14). Fix $q: \mathrm{Q} \rightarrow \mathrm{P}$. The monad $\bar{q}: \bar{Q} \rightarrow \bar{P}$ corresponds (under the equivalence of 2.1.3) to the presheaf

$$
\begin{aligned}
\mathrm{el}(\overline{\mathrm{P}})^{\mathrm{op}} & \longrightarrow \text { Set } \\
{[s: \mathrm{n} \rightarrow \overline{\mathrm{P}}] } & \longmapsto \text { Hom }_{\text {PolyEnd } / \overline{\mathrm{P}}(s, \bar{q}) .}
\end{aligned}
$$

But the required bijection,

$$
\operatorname{Hom}_{\text {PolyEnd } / \mathrm{P}}\left(E_{\mathrm{P}}(s), q\right) \simeq \operatorname{Hom}_{\text {PolyEnd } / \overline{\mathrm{P}}}(s, \bar{q})
$$

is precisely the factorisation property established in Lemma 2.2.4: in a diagram

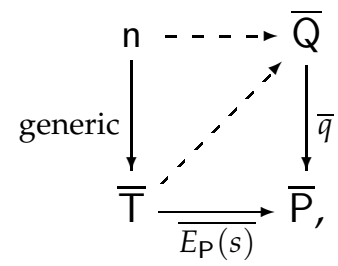

giving the top arrow $s \rightarrow \bar{q}$ (i.e. a map $n \rightarrow \bar{Q}$ over $\bar{P}$ ) is equivalent to giving the diagonal filler $E_{\mathrm{P}}(s) \rightarrow q$ (i.e. a map $\mathrm{T} \rightarrow \mathrm{Q}$ over $\mathrm{P}$ ).

2.2.7 Proposition. Let $\mathrm{T}$ be a tree and $\mathrm{P}$ an arbitrary polynomial endofunctor. Every monad map $h: \overline{\mathrm{T}} \rightarrow \overline{\mathrm{P}}$ factors essentially uniquely as

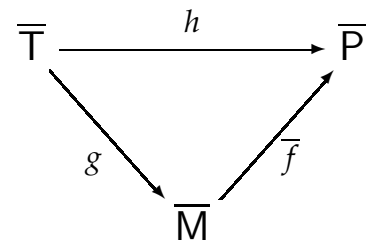

where $\mathrm{M}$ is a tree, $g: \overline{\mathrm{T}} \rightarrow \overline{\mathrm{M}}$ is boundary preserving (hence generic), and $\bar{f}: \overline{\mathrm{M}} \rightarrow \overline{\mathrm{P}}$ is free. 
Proof. Consider the map $h^{1}: \operatorname{sub}(\mathrm{T}) \rightarrow \operatorname{tr}(\mathrm{P})$ and let $f: \mathrm{M} \rightarrow \mathrm{P}$ be the image of $\mathrm{T} \in \operatorname{sub}(\mathrm{T})$. If $\mathrm{T}$ is the trivial tree, $h$ is already free, and $\mathrm{M}=\mathrm{T}$. Otherwise we construct $g: \overline{\mathrm{T}} \rightarrow \overline{\mathrm{M}}$ by grafting: each one-node subtree $\mathrm{S} \in \operatorname{sub}(\mathrm{T})$ is mapped by $h^{1}$ to some $\mathrm{P}$-tree $\mathrm{R}:=h^{1}(\mathrm{~S})$, and there is a unique boundary-preserving tree map $\overline{\mathrm{S}} \rightarrow \overline{\mathrm{R}}$. The map $g$ is the grafting of all these maps (indexed by the inner edges of T): since $h$ as a monad map preserves grafting, the grafting of the $P$-trees $R$ is precisely $M$. Uniqueness of the factorisation follows from Lemma 2.2.2.

2.2.8 Corollary. If $\mathrm{T}$ is a tree and $g: \overline{\mathrm{T}} \rightarrow \overline{\mathrm{R}}$ is generic in PolyEnd (with respect to the free-monad monad $\mathrm{P} \mapsto \overline{\mathrm{P}}$ ), then $\mathrm{R}$ is a tree and $\mathrm{g}$ is boundary preserving.

Proof. Factor $g$ as boundary preserving followed by free: $\bar{T} \rightarrow \bar{M} \rightarrow \bar{R}$, where $T$ is a tree. Since boundary-preserving maps between trees are generic, and by uniqueness of generic factorisations, we have $M \simeq R$, hence $g$ is boundary preserving.

2.2.9 Corollary. In the category Tree, the generic maps are precisely the boundary-preserving maps.

2.2.10 Proposition. The subcategory $\mathbf{t E m b} \subset$ PolyEnd provides arities for the free-monad monad F : PolyEnd $\rightarrow$ PolyEnd.

Proof. We have already shown that the free-monad monad is a local right adjoint, and that the subcategory $\mathbf{t E m b} \subset \boldsymbol{P} \boldsymbol{P o l y E n d}$ is (small and dense and) closed under generic factorisation. It remains to establish that the left Kan extension

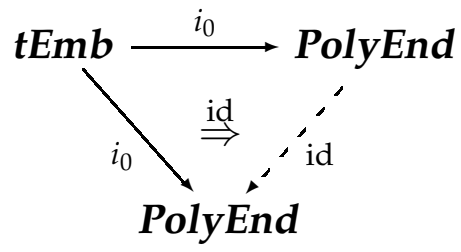

is preserved by the composite

$$
\text { PolyEnd } \stackrel{F}{\longrightarrow} \text { PolyEnd } \stackrel{N_{0}}{\longrightarrow} \operatorname{PrSh}(\boldsymbol{t E m b}) .
$$

We will show it is a pointwise extension, i.e. that for each $\mathrm{P} \in$ PolyEnd, the left Kan extension

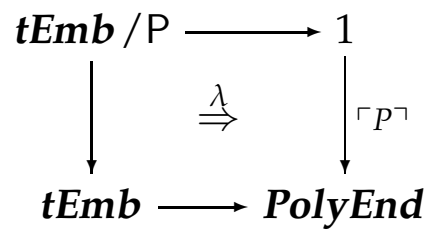

is preserved by $N_{0} \circ F$.

The claim is that (for fixed $X \in \boldsymbol{P r S h}(\boldsymbol{t} \operatorname{Em} \boldsymbol{b})$ ) each natural transformation

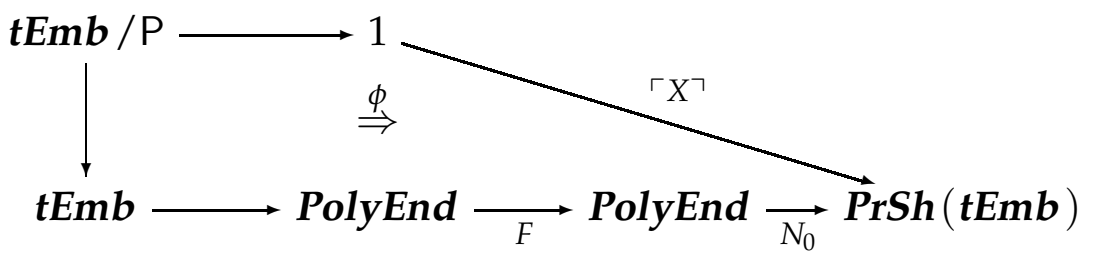


factors uniquely as

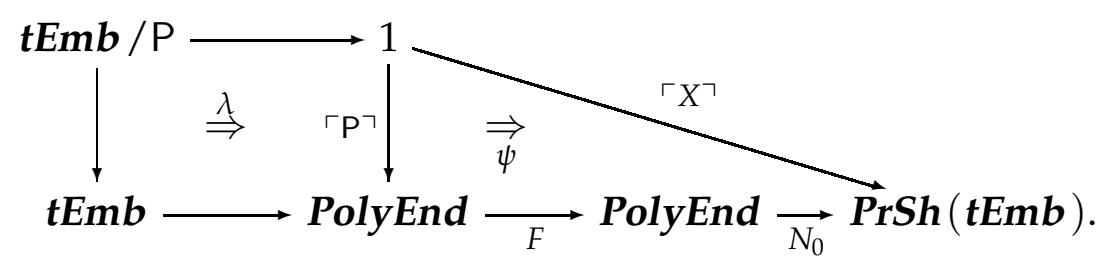

The component of $\phi$ at a P-tree $a: \mathrm{A} \rightarrow \mathrm{P}$ is a map of presheaves $\phi_{a}: \overline{\mathrm{A}} \rightarrow X$, i.e. for each (abstract) tree $T$ a natural map

$$
\phi_{a, \mathrm{~T}}: \operatorname{PolyEnd}(\mathrm{T}, \overline{\mathrm{A}}) \rightarrow X(\mathrm{~T}) .
$$

To specify $\psi$ we need for each tree $T$ a natural map

$$
\psi_{\mathrm{T}}: \operatorname{PolyEnd}(\mathrm{T}, \overline{\mathrm{P}}) \rightarrow X(\mathrm{~T}) .
$$

Finally, the component of $N_{0} \circ F \circ \lambda$ at a P-tree $a: \mathrm{A} \rightarrow \mathrm{P}$ and an abstract tree $\mathrm{T}$ is

$$
\begin{aligned}
\operatorname{PolyEnd}(\mathrm{T}, \overline{\mathrm{A}}) & \longrightarrow \text { PolyEnd }(\mathrm{T}, \overline{\mathrm{P}}) \\
z & \longmapsto \bar{a} \circ z .
\end{aligned}
$$

Now the key point is that every $f \in \operatorname{PolyEnd}(\mathrm{T}, \overline{\mathrm{P}})$ is in the image of this map for a suitable $a$ : factor $f$ into generic followed by free:

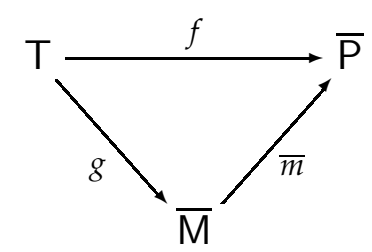

then

$$
f=\left(N_{0} \circ F \circ \lambda\right)_{m, T}(g) .
$$

So if $\psi$ is going to give $\phi$ after pasting with $\lambda$ we are forced to define

$$
\psi_{\mathrm{T}}(f):=\phi_{m, \mathrm{~T}}(g) \in X(\mathrm{~T}) .
$$

It is a routine calculation to verify that this assignment is natural in $T$. It relies on two facts: first, that generic-free factorisation is functorial: given $T^{\prime} \rightarrow T$ over $\bar{P}$ there is induced a unique $\mathrm{M}^{\prime} \rightarrow \mathrm{M}$ between the factorisations, and second, that $\phi$ is natural in M.

2.2.11 Remark. The above arguments are analogous to those of Weber [21], Prop.4.22, and they serve in fact to prove the following general result: let $F$ be a monad on an arbitrary category $\mathscr{C}$, and let $i_{0}: \Theta_{0} \rightarrow \mathscr{C}$ be fully faithful and dense (with $\Theta_{0}$ small). If the morphism $i_{0} \downarrow F i_{0} \downarrow F \longrightarrow i_{0} \downarrow F$ of categories fibred over $\Theta_{0}$ has connected fibres and admits a section (over $\Theta_{0}$ ) then $\Theta_{0}$ provides $F$ with arities. 
With Proposition 2.2.10 we are in position to apply Weber's general nerve theorem (2.0.5) directly, establishing this:

2.2.12 Theorem. The nerve functor $N:$ PolyMnd $\rightarrow \operatorname{PrSh}($ tree) is fully faithful. A presheaf $X:$ tree $^{\mathrm{op}} \rightarrow$ Set is isomorphic to the nerve of a polynomial monad if and only if its restriction $j^{*} X: \mathbf{t} \mathbf{E m} \boldsymbol{b}^{\text {op }} \rightarrow$ Set is isomorphic to the nerve of a polynomial endofunctor.

\subsection{Sheaf conditions and nerve theorem for slices}

Since we have now characterised polynomial monads in terms of their underlying endofunctors, we should now proceed to characterise polynomial endofunctors among all presheaves on $\boldsymbol{t} E \mathbf{m} \boldsymbol{b}$.

Since every object in PolyEnd is a colimit of elementary trees (in a canonical way), the embedding elTr $\rightarrow$ PolyEnd is dense, and therefore also $\mathbf{t E m b} \rightarrow$ PolyEnd is dense. This means that the nerve functor

$$
\begin{aligned}
N_{0}: \text { PolyEnd } & \longrightarrow \operatorname{PrSh}(\text { tEmb }) \\
\mathrm{P} & \longmapsto \operatorname{Hom}_{\text {PolyEnd }}(-, \mathrm{P})
\end{aligned}
$$

is fully faithful.

2.3.1. Grothendieck topology on $T E m b$. There is a Grothendieck topology on TEmb (and on $t E m b$ ): a family of tree embeddings $\left\{S_{\lambda} \rightarrow T\right\}_{\lambda \in \Lambda}$ is declared covering if it is jointly surjective on nodes, and also on edges. This topology has a more conceptual characterisation: the inclusion functor elTr $\rightarrow \boldsymbol{t E m b}$ induces a geometric morphism

$$
\operatorname{PrSh}(\text { elTr }) \rightarrow \operatorname{PrSh}(t E m b)
$$

which turns out to be a left exact localisation, hence defines a Grothendieck topology on $\boldsymbol{t} \mathbf{E m} \boldsymbol{b}$ - which is the one just described - inducing an equivalence

$$
\operatorname{PrSh}(\mathrm{elTr}) \stackrel{\sim}{\rightarrow} \operatorname{Sh}(\mathrm{tEmb}) .
$$

2.3.2. Reduced covers and generic injections. A covering family on a tree $T$ is called reduced if each node of $\mathrm{T}$ is only in one member of the family and if no member can be removed without spoiling the covering property. Another characterisation of reduced covers is: each outer edge of $\mathrm{T}$ is hit exactly once, and each inner edge is hit either once or twice. The reduced covers of $T$ form a poset $(\operatorname{Red} \operatorname{Cov}(T), \leq)$ with $F \leq G$ if the cover $F$ is a refinement of $G$. If $T$ is a nontrivial tree, there are isomorphisms of posets

$$
\operatorname{Red} \operatorname{Cov}(T)^{\mathrm{op}} \simeq \mathscr{P}\left(T^{1} \times_{T^{0}} T^{2}\right) \simeq \operatorname{GenInj}(T) .
$$

Here the middle poset is the powerset of the set of inner edges in T, and GenInj(T) denotes the poset of isomorphism classes of generic injections into $T$. The left-hand correspondence is: for a subset $J$ of inner edges, the reduced cover is given by cutting 
the tree at the inner edges in $J$. In other words, the inner edges in $J$ are those inner edges hit twice by the cover. For the right-hand correspondence, the inner edges in $J$ correspond to the inner edges that are hit by a generic injection. In the correspondence between reduced covers and generic injections, the covering condition corresponds to the generic condition (boundary preservation), while the reducedness of the cover corresponds to injectivity of the generic map.

2.3.3. The Segal condition. Each tree is canonically the colimit of its elements, i.e. the colimiting cone of its canonical diagram, cf. 2.1.7. A presheaf $X:$ tree $^{\mathrm{op}} \rightarrow$ Set is said to satisfy the Segal condition if $X$ sends these cocones in tree to limit cones in Set. The cocones in turn are just iterated pushouts of grafting type, so we can also state the Segal condition as the requirement of sending those pushouts to pullbacks. Finally, the canonical diagrams correspond to the minimal covering families (those for which all members are elementary trees), and since these form a basis for the topology, we can also say that $X$ satisfies the Segal condition if and only if it its restriction to $t \boldsymbol{E m} \boldsymbol{b}$ is a sheaf.

2.3.4 Proposition. If $\mathrm{P}$ is a polynomial endofunctor, then $N_{0} \mathrm{P}$ is a sheaf.

Proof. This follows directly from the fact that the pushouts of 1.1 .19 are also pushouts in the category PolyEnd. (Given $\mathrm{T}=\mathrm{S}+\mathrm{R}$, then

$$
\text { PolyEnd }(T, P) \longrightarrow \text { PolyEnd }(S, P) \times_{\text {PolyEnd }(1, P)} \text { PolyEnd }(R, P)
$$

is an isomorphism by the pushout property.)

With this result, we have factored the nerve functor $N_{0}$ as

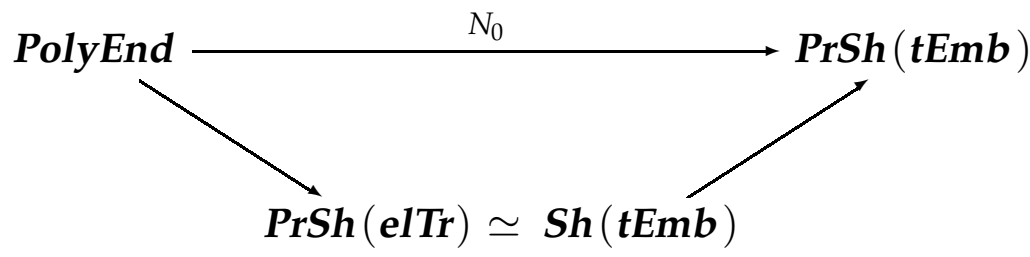

and reduced the question to that of characterising polynomial endofunctors among presheaves on elTr. Before dealing with this (in the next section), a remark is due on the sliced case.

2.3.5. Nerve theorem for slices. There is a pushout theorem for TEmb/P and PolyEnd/P in analogy with 1.1.19, and there is induced a Grothendieck topology on $T E \mathbf{m b} / \mathrm{P}$ giving an equivalence of categories

$$
\operatorname{PrSh}(\mathrm{elTr} / \mathrm{P}) \simeq \operatorname{Sh}(\boldsymbol{t E m b} / \mathrm{P}) .
$$

Let now $\mathrm{P}$ be a polynomial monad. Composition of functors makes PolyEnd / P a 2category; its monads are naturally identified with monads over P. In fact, PolyEnd / P 
is the category of P-collections, and its monads are the P-operads, in the sense of Leinster [14, §4.2]. Again, the forgetful functor PolyMnd/P $\rightarrow$ PolyEnd/P has a left adjoint which can be described in terms of maps from P-trees in analogy with 1.2.8. This yields the free P-monad monad which is in fact cartesian. (For fixed set of objects, this is proved in Leinster's book [14, §C.1].) Furthermore, this monad is a local right adjoint, as it follows from the arguments in 2.2.6. (Since PolyEnd /P is a presheaf category the notion of local right adjoint is equivalent to the notion of familially representable, and the result can also be extracted from [14, §C.3].)

We have seen that every element $\mathrm{n} \rightarrow \mathrm{P}$ factors through a tree, and that all $\mathrm{P}$-trees arise like this. The following result is now a direct application of the special nerve theorem (2.0.3).

2.3.6 Theorem. For a presheaf $X:(\text { tree/P })^{\text {op }} \rightarrow$ Set, the following are equivalent:

1. $X$ is in the essential image of $N$ (i.e. $X$ is isomorphic to the nerve of a polynomial monad over P).

2. $j^{*} X$ is in the essential image of $N_{0}$ (i.e. $j^{*} X$ is isomorphic to the nerve of a polynomial endofunctor over $\mathrm{P})$.

3. $j^{*} X$ is a sheaf on $\boldsymbol{t} \mathbf{E m b} / \mathrm{P} \simeq \operatorname{tr}(\mathrm{P})$.

4. X satisfies the Segal condition, i.e. takes the canonical cocones to limit cones.

The key point to note is that we have an equivalence of categories

$$
\text { PolyEnd } / \mathrm{P} \simeq \boldsymbol{S h}(\operatorname{tr}(\mathrm{P})) .
$$

2.3.7. Examples. If $P$ is the identity monad, we recover the classical nerve theorem for categories. For $P=M$ (the free-monoid monad), tree $/ M$ is the category of planar trees, polynomial monads over $\mathrm{M}$ are nonsymmetric operads, and the theorem says that such are characterised among presheaves on tree/M by the Segal condition.

\subsection{Polynomial endofunctors and collections}

2.4.1. Collections. The category $\operatorname{PrSh}(\mathbf{e l T r})$ is the category of (coloured, symmetric) collections, which we denote by Coll. To be explicit, a collection $C$ consists of a set $C(1)$ of colours and for each $n \in \mathbb{N}$ a set $C(n)$ of $n$-ary operations. The structure maps are first of all $n+1$ projections $C(n) \rightarrow C(1)$, and for each $n \in \mathbb{N}$ the symmetric group $\mathfrak{S}_{n}$ acts on $C(n)$ by permuting the first $n$ projections. The inverse image in $C(n)$ of the elements $\left(i_{1}, \ldots, i_{n} ; i\right) \in C(1)^{n+1}$ is denoted $C\left(i_{1}, \ldots, i_{n} ; i\right)$ and is interpreted as the set of $n$-ary operations with input colours $i_{1}, \ldots, i_{n}$ and output colour $i$.

Since elTr is dense in PolyEnd, the nerve functor

$$
\begin{aligned}
R_{0}: \text { PolyEnd } & \longrightarrow \operatorname{PrSh}(\text { elTr })=\text { Coll } \\
\mathrm{P} & \longmapsto \operatorname{Hom}(-, \mathrm{P})
\end{aligned}
$$


is fully faithful. We proceed to characterise its image, and start by looking at the slices:

2.4.2 Proposition. The nerve functor $R_{0}:$ PolyEnd $\rightarrow \operatorname{PrSh}(\mathbf{e l T r})$ is a local equivalence. That is, for every polynomial endofunctor $P$, the sliced functor

$$
\begin{aligned}
\text { PolyEnd } / \mathrm{P} & \longrightarrow \operatorname{PrSh}(\text { elTr }) / R_{0} \mathrm{P} \\
{[\mathrm{Q} \rightarrow \mathrm{P}] } & \longmapsto\left[R_{0} \mathrm{Q} \rightarrow R_{0} \mathrm{P}\right]
\end{aligned}
$$

is an equivalence.

Proof. We use the equivalence PolyEnd $/ \mathrm{P} \simeq \boldsymbol{P r S h}(\mathrm{el}(\mathrm{P})) \simeq \boldsymbol{P r S h}(\mathbf{e l T r} / \mathrm{P})$ of Proposition 2.1.3 (with equation (13)). Under this equivalence the sliced nerve functor has the following description: it sends a presheaf $\widetilde{Q}:(\mathbf{e l T r} / \mathrm{P})^{\text {op }} \rightarrow$ Set to

$$
\begin{aligned}
X: \text { elTr }^{\text {op }} & \longrightarrow \text { Set } \\
\mathrm{n} & \longmapsto \sum_{s: \mathrm{n} \rightarrow \mathrm{P}} \widetilde{\mathrm{Q}}(s)
\end{aligned}
$$

(This presheaf has a natural map to $R_{0} \mathrm{P}=[\mathrm{n} \mapsto \operatorname{Hom}(\mathrm{n}, \mathrm{P})]$ by returning the running index of the sum.)

In the other direction, given a presheaf $X: \mathbf{e l T r}{ }^{\mathrm{op}} \rightarrow$ Set with a map $\alpha: X \Rightarrow R_{0} \mathrm{P}$, define

$$
\begin{aligned}
\widetilde{\mathrm{Q}}:(\mathbf{e l T r} / \mathrm{P})^{\mathrm{op}} & \longrightarrow \text { Set } \\
{[s: \mathrm{n} \rightarrow \mathrm{P}] } & \longmapsto X(\mathrm{n})_{s}
\end{aligned}
$$

where $X(\mathrm{n})_{s}$ denotes the fibre of $\alpha_{\mathrm{n}}: X(\mathrm{n}) \rightarrow \operatorname{Hom}(\mathrm{n}, \mathrm{P})$ over $s$.

It is easy to see that these two functors are inverse to each other, establishing the asserted equivalence.

2.4.3. The nerve and its slices. For each polynomial endofunctor $P$ we have a diagram

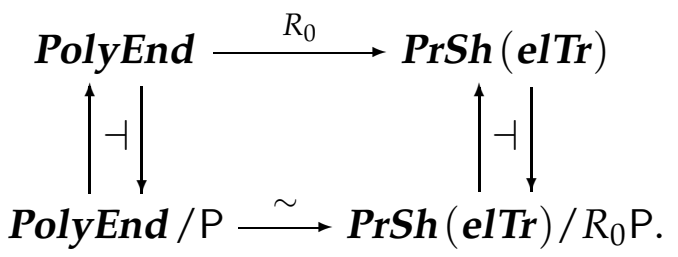

The left adjoints are just forgetting the structure map to $\mathrm{P}$ and $R_{0} \mathrm{P}$, respectively, and clearly the square with the left adjoints commutes. The right adjoints are multiplication with $\mathrm{P}$ and multiplication with $R_{0} \mathrm{P}$, respectively. The square with the right adjoints commutes because $R_{0}$, as every nerve functor, commutes with limits, and in particular with products.

The right adjoint on presheaves has another conceptual description, via the equivalence $\operatorname{PrSh}(\mathbf{e l T r}) / R_{0} \mathrm{P} \simeq \operatorname{PrSh}(\mathbf{e l T r} / \mathrm{P})$ : in terms of the latter it is just precomposition with the forgetful functor elTr $/ P \rightarrow$ elTr. 
2.4.4. Nonsymmetric collections. The above diagram is most interesting when $P$ is the free-monoid monad $\mathrm{M}$ : in this case we have

$$
\operatorname{PrSh}(\mathbf{e l T r}) / R_{0} \mathrm{M} \simeq \operatorname{PrSh}(\mathrm{el}(\mathrm{M})),
$$

and the latter is the category of nonsymmetric collections, denoted NonSymColl, and the left adjoint is then the symmetrisation functor, denoted $S$. The category el(M) is equivalent to the category of planar elementary trees. Nonsymmetric collections are described just as collections, except that there are no symmetries.

The diagram now reads:

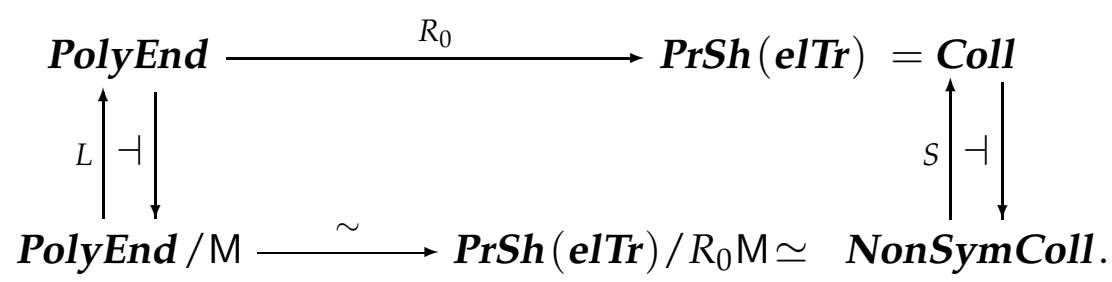

Note that $L$ is surjective on objects. Indeed, every polynomial endofunctor admits a map to M. It follows that $R_{0}$ and $S$ have the same essential image. Since $R_{0}$ is fully faithful we get:

2.4.5 Theorem. The category PolyEnd, as a subcategory of Coll, is naturally identified with the Kleisli category for the symmetrisation monad S on NonSymColl.

2.4.6 Remark. The symmetrisation monad $S$ on $\operatorname{PrSh}(\mathbf{e l T r} / \mathrm{M})$ is a local right adjoint, since it is the composite of a forgetful functor from a slice category and a true right adjoint. It is endowed with arities by the representables themselves, and elTr appears as the Kleisli category of elTr/M with respect to $S$. The generic/free factorisation on elTr is quite degenerate: every arrow in elTr already is either a generic map (an automorphism of some $n$ ) or it is free (an inclusion $1 \rightarrow n$ ).

Slightly more interesting is the corresponding generic/free factorisation system on Tree, the Kleisli category on the category Tree / M, still with respect to $S$. To see it most clearly, let ptree denote a skeleton of Tree/M, the category of planar trees. Let tree' denote the full subcategory of Tree with one object for each object in ptree (i.e. the category of planar trees and not-necessarily planar maps). This is just the Kleisli category of $S$ restricted to ptree. Now in tree $^{\prime}$, the generics are the isomorphisms and the free maps are the planar maps, and every arrow factors as an isomorphism followed by a planar map (in analogy with the skeleton of the category of finite sets consisting of the sets $\{0, \ldots, n-1\}$ which happen to have a natural order: every arrow in this category factors as an isomorphism followed by an order-preserving map).

2.4.7. Polynomial endofunctors as flat collections. There is another characterisation of PolyEnd as a subcategory of Coll, suggested by André Joyal: it is the subcategory of 'projective' objects with respect to a certain class of surjections. Call a collection $P$ flat if every colour-preserving surjection $Z \rightarrow P$ admits a section. 
2.4.8 Theorem. A collection $P$ is isomorphic to the nerve of a polynomial endofunctor if and only if it is flat.

Theorem 2.4.8 will be broken into a chain of biimplications (Theorem 2.4.10) each of which is rather easy to establish, once the correct viewpoint has been set up.

2.4.9. Collections with a fixed set of colours. We denote by Coll $(I)$ the category of collections with colour set $I$ and colour preserving morphisms. This category is again (equivalent to) a presheaf category. Namely, let MonEnd (I) be the full subcategory of PolyEnd $(I)$ consisting of the monomial endofunctors (with endpoints $I$ ), i.e. those $I \leftarrow$ $E \rightarrow B \rightarrow I$ for which $B$ is singleton. Clearly MonEnd $(I)$ is a groupoid. Write $n$ for a fixed $n$-element set, and denote by monEnd $(I) \subset \operatorname{MonEnd}(I)$ the small subgroupoid consisting of the objects

$$
I \leftarrow n \rightarrow 1 \rightarrow I
$$

$(n \in \mathbb{N})$. It is a disjoint union:

$$
\operatorname{monEnd}(I)=\sum_{n \in \mathbb{N}} \operatorname{monEnd}(I)_{n}
$$

where monEnd $(I)_{n}$ is the subgroupoid of monomials of degree $n$. Now we have

$$
\operatorname{Coll}(I) \simeq \operatorname{PrSh}(\text { monEnd }(I)) .
$$

(This viewpoint on Coll (I) was also used in the appendix of Berger-Moerdijk [4], except that they did not formulate it in terms of monomial functors.) An object $I \leftarrow n \rightarrow$ $1 \rightarrow I$ amounts to an $(n+1)$-tuple $\left(i_{1}, \ldots, i_{n} ; i\right)$ of elements in $I$, and the value on it of a presheaf $C$ is the set $C\left(i_{1}, \ldots, i_{n} ; i\right)$; the arrows in monEnd $(I)$ provide the colourpreserving symmetries. More formally, given a presheaf $F:$ monEnd $(I)^{\mathrm{op}} \rightarrow \boldsymbol{S e t}$, define a presheaf with colour set $I$ by

$$
\begin{aligned}
\text { elTr }^{\mathrm{op}} & \longrightarrow \text { Set } \\
\mathrm{n} & \longmapsto \sum_{\mathrm{Q} \in \operatorname{monEnd}(I)_{n}} F(\mathrm{Q}),
\end{aligned}
$$

and conversely, given a presheaf $C: \mathbf{e l T r}{ }^{\mathrm{op}} \rightarrow$ Set with $C(1)=I$, define a presheaf on monEnd $(I)$ by sending an object $\left(i_{1}, \ldots, i_{n} ; i\right)$ to the inverse image of this $(n+1)$-tuple under the structure map $C(n) \rightarrow I$.

Since monEnd $(I)$ is a full subcategory of PolyEnd $(I)$ we have a nerve functor

$$
\begin{aligned}
R_{0(I)}: \text { PolyEnd }(I) & \longrightarrow \operatorname{PrSh}(\text { monEnd }(I)) \\
\mathrm{P} & \longmapsto \operatorname{Hom}_{\text {PolyEnd }(I)}(-, \mathrm{P}),
\end{aligned}
$$

and this nerve functor is compatible with the global nerve $R_{0}:$ PolyEnd $\rightarrow$ Coll. Precisely, the diagram 


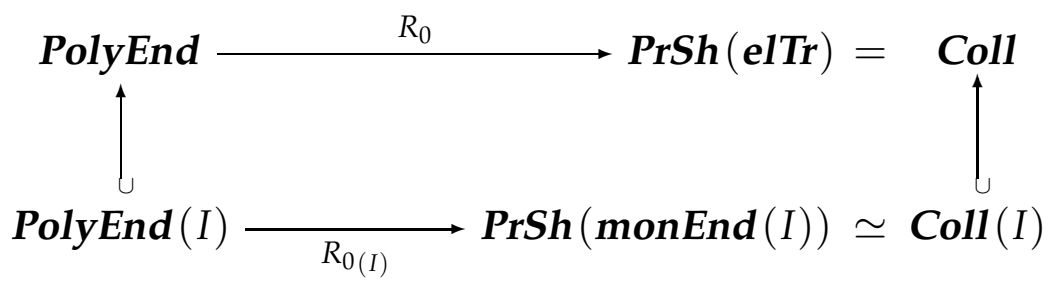

commutes up to a natural isomorphism, as is easy to check.

2.4.10 Theorem. For any collection $P$ with set of colours $I$, the following are equivalent.

1. P is flat (i.e. every colour-preserving surjection onto P in Coll admits a section).

2. $P$ is projective in Coll(I) (i.e. every surjection onto P in Coll(I) admits a section).

3. $P$ is a sum of representables in $\operatorname{Coll}(I) \simeq \operatorname{PrSh}(\operatorname{monEnd}(I))$.

4. $P$ is in the essential image of the nerve functor $R_{0(I)}:$ PolyEnd $(I) \rightarrow \operatorname{Coll}(I)$.

5. $P$ is in the essential image of the nerve functor $R_{0}:$ PolyEnd $\rightarrow$ Coll.

Proof. (1) $\Leftrightarrow(2)$ : obvious.

(2) $\Leftrightarrow(3)$ : It is true in any category of presheaves on a groupoid that an object is projective (with respect to termwise surjections) if and only if it is a sum of representables.

(3) $\Leftrightarrow(4)$ : The $R_{0(I)}$-nerve of a polynomial functor $I \leftarrow E \rightarrow B \rightarrow I$ is the sum of the representables $I \leftarrow E_{b} \rightarrow\{b\} \rightarrow I$ (indexed by $b \in B$ ). Conversely any sum of representables defines a polynomial endofunctor (with endpoints $I$ ). Phrased more elegantly: polynomial endofunctors are precisely the sums of monomial endofunctors.

$(4) \Leftrightarrow(5)$ : This follows from the fact that the nerve functors are compatible.

\subsection{Polynomial monads and operads}

In this final section we characterise the image of the nerve functor for polynomial monads, explain the relation with operads, and sum up the relation between the various nerve functors.

Combining Proposition 2.2.12 and Proposition 2.4.8, we already have:

2.5.1 Proposition. A presheaf $X:$ tree $^{\mathrm{op}} \rightarrow$ Set is isomorphic to the nerve of a polynomial monad if and only if $j^{*} X: \mathbf{t E m} \boldsymbol{b}^{\text {op }} \rightarrow$ Set is a flat collection. 
We now finally come to operads, and aim to fit everything into this diagram:

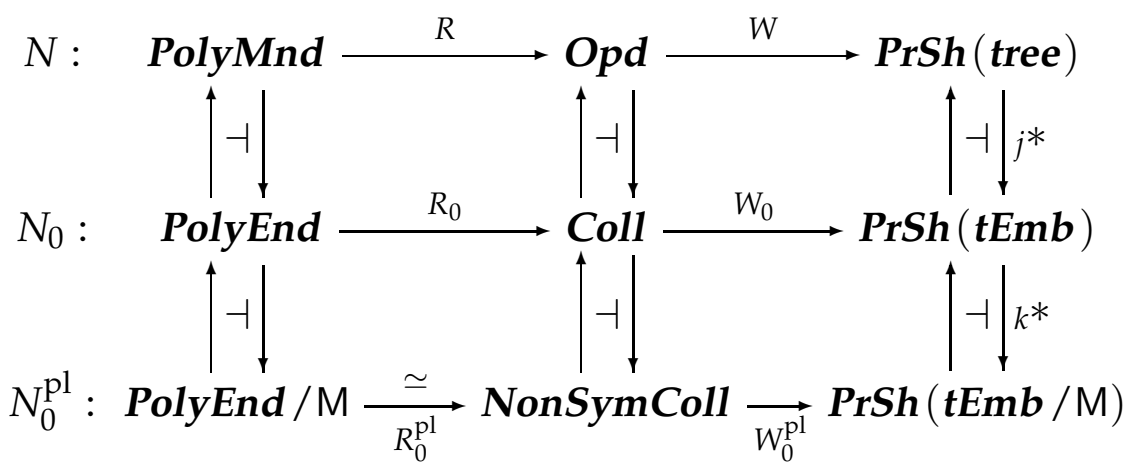

The middle row and the bottom row were explained in the previous section.

2.5.2. Operads. For fixed set of colours $I$, the category Coll $(I)$ has a monoidal structure given by the substitutional tensor product (cf. [11], see also the Appendix of [4]). The monoids in Coll (I) are the I-coloured operads, forming a category $\mathbf{O p d}(I)$ and fitting into a free-forgetful adjunction $\operatorname{Opd}(I) \rightleftarrows$ Coll $(I)$. Morphisms between operads of different colours can be defined in terms of base-change (just as for collections): if $\alpha: I \rightarrow J$ is a map of sets there is an obvious change-of-colours functor $\alpha^{*}: \operatorname{Opd}(J) \rightarrow \boldsymbol{O p d}(I)$, and a morphism from an I-coloured operad $X$ to a $J$-coloured operad $Y$ is defined to be a pair $(\alpha, \phi)$ where $\alpha: I \rightarrow J$ is a map of sets and $\phi: X \rightarrow \alpha^{*} Y$ is a morphism of $I$-coloured operads. With this extra structure the free-forgetful adjunctions assemble into a single adjunction

\section{Opd $\rightleftarrows$ Coll}

The functor $R_{0}:$ PolyEnd $\rightarrow$ Coll is monoidal (for each $I$ ) and commutes with base-change, hence induces the functor

$$
R: \text { PolyMnd } \rightarrow \text { Opd } .
$$

2.5.3. The nerve functor for operads. The nerve functor $W:$ Opd $\rightarrow \operatorname{PrSh}($ tree $)$ is now defined in the obvious way from the embedding tree $\subset$ PolyMnd $\subset$ Opd. It is the nerve functor for operads introduced by Moerdijk and Weiss. The image of $W$ is characterised in the following theorem, due to Moerdijk-Weiss and Weber:

2.5.4 Theorem. For a presheaf $X:$ tree $^{\mathrm{op}} \rightarrow$ Set, the following are equivalent.

1. $X$ is isomorphic to the nerve of an operad (i.e. $X$ is in the essential image of W).

2. Every inner horn of $X$ has a unique filler.

3. $j^{*} X$ is isomorphic to the nerve of a collection (i.e. $j * X$ is in the essential image of $W_{0}$ ).

4. $k^{*} j^{*} X$ is isomorphic to the nerve of a nonsymmetric collection (i.e. $k^{*} j^{*} X$ is in the essential image of $W_{0}^{p l}$ ). 
5. X satisfies the Segal condition (i.e. takes canonical cocones to limit cones.)

Proof. The equivalence of (1) and (2) is due to Moerdijk and Weiss [18] (their Proposition 5.3 together with Theorem 6.1). We shall not need the horn-filling condition here, and mention the result only because it was the first nerve theorem for operads. The equivalence of (1), (4) and (5) are due to Weber [21] (combining his Examples 2.14, 4.19, and 4.27). Condition (3) and the category $\mathbf{t E m b}$, central to the present paper, are not considered by Moerdijk and Weiss, nor by Weber. The equivalence between (4) and (3) follows from the fact that the symmetrisation functor is a local right adjoint on a presheaf category (cf. 2.4.6).

For emphasis, we restate Proposition 2.2.12 as a characterisation of polynomial monads among operads:

2.5.5 Proposition. An operad is isomorphic to a polynomial monad if and only if its underlying collection is flat.

Acknowledgments. I am especially indebted to André Joyal for his contributions to my mathematical apprenticeship, and specifically for introducing me to polynomial functors. Countless discussions with him on the subject over the past years have been crucial for this work. This work was prompted by Ieke Moerdijk's talk on dendroidal sets at the Mac Lane Memorial Conference in April 2006 - I am grateful for the input. The first part of the paper was in place in March 2007 when I presented it at the 85th Peripatetic Seminar on Sheaves and Logic in Nice. At that occasion Clemens Berger pointed out the similarities with his theory [3] of Joyal's Theta [9] and pointed me to Weber's work [20], [21]; many conceptual improvements resulted from these links, and the second part of the paper found its natural setting - I thank him for those pointers. More recently I have profited from conversations with Anders Kock, David Gepner, Denis-Charles Cisinski, Ieke Moerdijk, Mark Weber, Michael Batanin, Nicola Gambino, and Tom Fiore, all of whom have been visiting Barcelona to participate in the special programme on Homotopy Theory and Higher Categories at the CRM, where this work was finished. I am grateful to Panagis Karazeris for organising the Patras conference (March 2008) in honour of my father, the perfect opportunity for me to present this work. Finally I acknowledge benefit from research grants MTM2006-11391 and MTM2007-63277 of the Spanish Ministry of Education and Science.

\section{References}

[1] John C. BAEZ and JAMES Dolan. Higher-dimensional algebra. III. n-categories and the algebra of opetopes. Adv. Math. 135 (1998), 145-206. ArXiv:q-alg/9702014. 
[2] Michael BARr and Charles Wells. Toposes, triples and theories. No. 278 in Grundlehren der Mathematischen Wissenschaften. Springer-Verlag, 1985. Corrected reprint in Reprints in Theory and Applications of Categories, 12 (2005) (electronic).

[3] Clemens Berger. A cellular nerve for higher categories. Adv. Math. 169 (2002), 118-175.

[4] Clemens Berger and IeKe MoerdijK. Resolution of coloured operads and rectification of homotopy algebras. In Categories in algebra, geometry and mathematical physics, vol. 431 of Contemp. Math., pp. 31-58. Amer. Math. Soc., Providence, RI, 2007. ArXiv:math/0512576.

[5] Nicola Gambino and Martin Hyland. Wellfounded Trees and Dependent Polynomial Functors. In S. Berardi, M. Coppo, and F. Damiani, editors, TYPES 2003, vol. 3085 of Lecture Notes in Computer Science, pp. 210-225. Springer Verlag, Heidelberg, 2004.

[6] Nicola Gambino and JoAchim Kock. Polynomial functors and polynomial monads. Preprint, arXiv:0906.4931.

[7] Victor Ginzburg and Mikhail Kapranov. Koszul duality for operads. Duke Math. J. 76 (1994), 203-272. ArXiv:0709.1228.

[8] ANDRÉ JOYAL. Foncteurs analytiques et espèces de structures. In Combinatoire énumérative (Montréal/Québec, 1985), vol. 1234 of Lecture Notes in Math., pp. 126159. Springer, Berlin, 1986.

[9] André Joyal. Disks, duality and @-categories. Preprint, September 1997.

[10] ANDRÉ JOYAL and JOACHIM KOCK. Feynman graphs, and nerve theorem for compact symmetric multicategories (extended abstract). To appear in the proceedings of "Quantum Physics and Logic VI", Oxford, 2009. Electronic Notes in Theoretical Computer Science. ArXiv:0908.2675.

[11] G. Max Kelly. On the operads of J. P. May. Repr. Theory Appl. Categ. (2005), 1-13 (electronic). Written 1972.

[12] JoAсHIM KосK. Notes on polynomial functors. Rough draft, 420pp. Available from http://mat.uab.cat/ kock/cats/polynomial.html.

[13] JoAchim Kock, André Joyal, Michael Batanin, and Jean-François MasCARI. Polynomial functors and opetopes. Preprint, arXiv:0706.1033.

[14] TOM Leinster. Higher Operads, Higher Categories. London Math. Soc. Lecture Note Series. Cambridge University Press, Cambridge, 2004.

ArXiv:math.CT/0305049. 
[15] TOM LeINSTER. Nerves of algebras. Talk at CT04, Vancouver. Slides available from http://www.maths.gla.ac.uk/ tl/vancouver/.

[16] IeKe MoerdijK and Erik Palmgren. Wellfounded trees in categories. Annals of Pure and Applied Logic 104 (2000), 189-218.

[17] IeKe MoerdiJK and ItTAy Weiss. Dendroidal sets. Alg. Geom. Top. (2007), 14411470. ArXiv:math/0701293.

[18] IEKe MOeRDiJK and ItTAy WeISS. On inner Kan complexes in the category of dendroidal sets. Adv. Math. 221 (2009), 343-389. ArXiv:math/0701295.

[19] Ross Street. The petit topos of globular sets. J. Pure Appl. Algebra 154 (2000), 299-315. Category theory and its applications (Montreal, QC, 1997).

[20] MARK WEBER. Generic morphisms, parametric representations and weakly Cartesian monads. Theory Appl. Categ. 13 (2004), 191-234 (electronic).

[21] MARK WEBER. Familial 2-functors and parametric right adjoints. Theory Appl. Categ. 18 (2007), 665-732 (electronic).

Joachim Kock <kock@mat . uab. cat >

Departament de matemàtiques

Universitat Autònoma de Barcelona

08193 Bellaterra (Cerdanyola), Spain 